
\title{
BResarch S Suare \\ Pitchfork and Hopf Bifurcations of Geared Systems in Permanent Contact Regime
}

\section{Mohsen Azimi ( $\square$ azimimohsen@email.arizona.edu )}

The University of Arizona https://orcid.org/0000-0002-0388-1873

\section{Research Article}

Keywords: System of nonlinear coupled differential equations, primary parametric resonance, combined parametric resonance, pitchfork bifurcation, hopf bifurcation

Posted Date: May 24th, 2021

DOl: https://doi.org/10.21203/rs.3.rs-511902/v1

License: (9) This work is licensed under a Creative Commons Attribution 4.0 International License. Read Full License

Version of Record: A version of this preprint was published at Nonlinear Dynamics on January 22nd, 2022. See the published version at https://doi.org/10.1007/s11071-021-07110-x. 


\title{
Pitchfork and Hopf Bifurcations of Geared Systems in Permanent Contact Regime
}

\author{
Mohsen Azimi
}

Received: date / Accepted: date

\begin{abstract}
Gears are known as important mechanical parts with various industrial applications. Many researchers investigated the complex nonlinear behavior of the geared systems by studying the effect of the clearance between the gears in mesh. This is while some studied the effect of both nonlinear suspension and clearance on nonlinear characteristics of such systems. Most of these studies are under assumption that the system operates under lightly loaded operational condition, where the separation of the teeth in mesh occurs. Alternatively, in this work, it is assumed that the transmitting load is big enough that gears in mesh do not separate and consequently the clearance between the teeth do not participates in the dynamic response of the system. Then analytical and numerical techniques are used to investigate the effect of nonlinear suspension on the dynamic behavior of the system. The results show that nonlinearity of the suspension has a great influence on the creation of the nontrivial equilibria or limit cycle within the unstable regions, which for the right range of the parameters, can affect the rate of the amplitude detonation and stabilization of the system.
\end{abstract}

Keywords System of nonlinear coupled differential equations · primary parametric resonance $\cdot$ combined parametric resonance $\cdot$ pitchfork bifurcation . hopf bifurcation

M. Azimi

Department of Aerospace and Mechanical Engineering, The University of Arizona, Tucson, AZ 85721, USA

Tel.: +1 (520) 6262053

E-mail: azimimohsen@email.arizona.edu 


\section{Introduction}

Gear transmission systems are crucial components with a wide range of applications which usually operate under complex conditions like high speed, heavy load, and variable torque. There has been various research on the dynamic analysis of the gears to identify the parameters that ensure the stability of the system under different operational conditions. This is mainly to avoid severe vibrations, damage, break done of the machinery, and catastrophic failure. It is well known that the tribological characteristics of the gears in mesh contributes a great influence on the dynamic response of the system. Such that the time varying meshing stiffness cause parametric excitation and consequently parametric resonance and backlash introduce strong nonlinearity to the systems. In different articles, several mathematical models are proposed to analyze the dynamic behaviors of different types of gears. For each of these cases, different techniques like phase plane, Poincare map [6], bifurcation diagram [5], and Lyapunov exponent [7] are used to study the complex dynamic characteristics of such systems. In most of these studies the nonlinear characteristics of the geared systems is analyzed under the assumption that the system operates under constant speed and lightly loaded operational condition. Where, the clearance between the gears are considered as the main source of the nonlinearity $[11,14]$. This is while, there are some works that analyzed the performance of the geared system by including the nonlinear effect of the supporting shaft $[2,13]$ or the clearance of the bearing $[6,3]$ along with the nonlinearity due to the backlash, which lead into occurrence of free play mode, and impact phases [8]. But not many attempts have been made to investigate the effect of the nonlinear suspension on the performance of the gears under the assumption that the gears in mesh do not separate and the nonlinearity due to free play mode and impact phases do not participate in system's response. This is a condition that can be acceptable under steady state operational conditions of the constant speed and high load [4], where the gears in mesh remain in permanent contact.

In our previous work, it was shown that by imposing the permanent contact condition to a system with linear suspension, the governing differential equations of the system reduces to a system of linear parametrically excited coupled differential equations. It was shown that for such a system the amplitude of the oscillation remain small until the parametric resonance happens, where the amplitude of the oscillation increases, and the permanent contact condition is violated. In such a condition the teeth in mesh separate and the free play mode and impact phases must be considered in the dynamic analysis of the system. Consequently, the goal of this paper is to impose the permanent contact condition to the same system but with nonlinear suspension and investigate the effect of this nonlinearity on the dynamic behavior of the system around the parametrically unstable tongues. To this end, the lumped parameter analysis method is used to drive the non-dimensional governing system of equations of a one-stage spur gear pair with nonlinear suspension. Then Poincare-Lindstedt method is used to investigate the influence of the parame- 
ters on the stability and bifurcation diagram of the system specifically around the unstable tongues. The results reveal that with the change of the control parameter, the meshing frequency, the system undergoes both pitchfork and hopf bifurcations around the primary and combined parametrically unstable tongues, respectively. At the end, numerical integration is used to investigate the Poincare map and time response of the nonlinear system for validating the analytical results. The analytical results are provided for both hardening and softening cubic nonlinearity of the suspension, but the numerical results is provided only for the softening case as the experimental data reported mostly softening behavior for such a system [1].

The remainder of this paper is organized as follows. In section 2, the nondimensional dynamic model of a spur gear pare with nonlinear suspension is provided. In section 3, Poincare-Lindstedt method is used to demonstrate the pitchfork and hopf bifurcation around the unstable tongues by studying the eigenvalues of the corresponding Jacobian matrix. In section 4, the results from numerical simulation are presented which demonstrate the behavior of the system under different operational conditions. Finally, in section 5, some brief conclusions and remarks are represented.

\section{Generalized Model of Spur Gear Pairs in Mesh}

In this section, the lumped parameter technique is used to formulate the dynamic model of a single-stage spur gear is mesh with nonlinear suspension. As illustrated in Fig. 1 the gears in mesh are modeled as a pair of rigid disks with radiuses equal to the base circles connected by a spring-damper set along the line of the action and the transmission shafts and the supporting mounts are modeled by a set of nonlinear spring and linear damper.

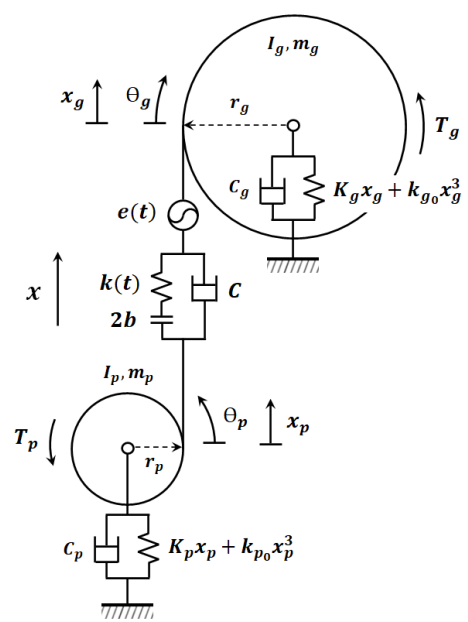

Fig. 1 Schematic of pinion and gear in mesh with nonlinear suspension 
For such a system, each disk has one rotational and one translational degree of freedom, consequently, the Newton's second law is used to construct the torsional and translational differential equation of motion for each disk.

$$
\begin{aligned}
M_{p} \ddot{x}_{p} & +C_{p} \dot{x}_{p}+K_{p} x_{p}+K_{p o} x_{p}^{3} \\
& +C\left(\dot{x}_{p}-\dot{x}_{g}+r_{p} \dot{\theta}_{p}-r_{g} \dot{\theta}_{g}-\dot{e}(t)\right) \\
& +k(t) f\left(x_{p}-x_{g}+r_{p} \theta_{p}-r_{g} \theta_{g}-e(t)\right)=0 \\
M_{g} \ddot{x}_{g} & +C_{g} \dot{x}_{g}+K_{g} x_{g}+K_{g o} x_{g}^{3} \\
& -C\left(\dot{x}_{p}-\dot{x}_{g}+r_{p} \dot{\theta}_{p}-r_{g} \dot{\theta}_{g}-\dot{e}(t)\right) \\
& -k(t) f\left(x_{p}-x_{g}+r_{p} \theta_{p}-r_{g} \theta_{g}-e(t)\right)=0 \\
I_{p} \ddot{\theta}_{p} & +r_{p} C\left(\dot{x}_{p}-\dot{x}_{g}+r_{p} \dot{\theta}_{p}-r_{g} \dot{\theta}_{g}-\dot{e}(t)\right) \\
& +r_{p} k(t) f\left(x_{p}-x_{g}+r_{p} \theta_{p}-r_{g} \theta_{g}-e(t)\right) \\
& =+\bar{T}_{p}+\widetilde{T}_{p} \cos \left(\omega_{p} t\right) \\
& \\
I_{g} \ddot{\theta}_{g} & -r_{p} C\left(\dot{x}_{p}-\dot{x}_{g}+r_{p} \dot{\theta}_{p}-r_{g} \dot{\theta}_{g}-\dot{e}(t)\right) \\
& -r_{g} k(t) f\left(x_{p}-x_{g}+r_{p} \theta_{p}-r_{g} \theta_{g}-e(t)\right) \\
& =-\bar{T}_{g}-\widetilde{T}_{g} \cos \left(\omega_{g} t\right)
\end{aligned}
$$

In these equations, subscripts $g$ and $p$ stand for gear and pinion, respectively. The total difference between the rotation angle of the gears in mesh can be expressed by the following equation which is defined as the difference between the dynamic and static transmission errors.

$$
x=x_{p}-x_{g}+r_{p} \theta_{p}-r_{g} \theta_{g}-e(t)
$$

Besides, for considering the fluctuation of the excitation toque and the applied load, both can be decomposed into averaging and fluctuating parts [5], so Eqs.(1)-(4) can be written in the following form.

$$
\begin{aligned}
& M_{p} \ddot{x}_{p}+C_{p} \dot{x}_{p}+K_{p} x_{p}+K_{p o} x_{p}^{3}+C \dot{x}+k(t) f(x)=0 \\
& M_{g} \ddot{x}_{g}+C_{g} \dot{x}_{g}+K_{p} x_{g}+K_{g o} x_{g}^{3}-C \dot{x}-k(t) f(x)=0 \\
& I_{p} \ddot{\theta}_{p}+r_{p} C x+r_{p} k(t) f(x)=+\bar{T}_{p}+\widetilde{T}_{p} \cos \left(\omega_{p} t\right) \\
& I_{g} \ddot{\theta}_{g}-r_{p} C x-r_{g} k(t) f(x)=-\bar{T}_{g}-\widetilde{T}_{g} \cos \left(\omega_{g} t\right)
\end{aligned}
$$

where $\bar{T}_{p}$ and $\bar{T}_{g}$ are the average torques and $\widetilde{T}_{p}$ and $\widetilde{T}_{g}$ are the fluctuating torques. By defining the following time varying gear mesh stiffness

$$
k(t)=K\left(1+k_{0} \cos (\omega t)\right), \quad k_{0}=\frac{k}{K}
$$


and subtracting Eq. (9) from Eq. (8), the governing torsional equations of motion reduce to the following equation.

$$
\begin{aligned}
-\ddot{x}_{p} & +\ddot{x}_{g}+\ddot{x}+\frac{C}{M} \dot{x}+\frac{K}{M}\left(1+k_{0} \cos (\omega t)\right) f(x)=\frac{K}{M} F \\
& +\frac{K}{M} F_{p} \cos \left(\omega_{p} t\right)+\frac{K}{M} F_{g} \cos \left(\omega_{g} t\right)-\ddot{e}(t)
\end{aligned}
$$

where $M$ is the equivalent mass representing the total inertia of the gear pair, $F$ is the average static force transmitted through the gear pair and $F_{p}$ and $F_{g}$ are the fluctuating force applied on the pinion and gear, expressed by the following equations.

$$
\begin{gathered}
M=\frac{I_{p} I_{g}}{I_{p} r_{g}^{2}+I_{g} r_{p}^{2}}, \quad F=\frac{M}{K}\left(\frac{\bar{T}_{p} r_{p}}{I_{p}}+\frac{\bar{T}_{g} r_{g}}{I_{g}}\right) \\
F_{p}=\frac{M}{K} \frac{\widetilde{T}_{p} r_{p}}{I_{p}}, \quad F_{g}=\frac{M}{K} \frac{\widetilde{T}_{g} r_{g}}{I_{g}}
\end{gathered}
$$

By defining the following standard parameters

$$
\omega_{n}=\sqrt{\frac{K}{M}}, \quad \zeta=\frac{C}{2 \sqrt{K M}}
$$

and the following static transmission error

$$
e(t)=E \cos (\omega t)
$$

one can write Eq. (11) in the following standard form.

$$
\begin{aligned}
-\ddot{x}_{p} & +\ddot{x}_{g}+\ddot{x}+2 \zeta \omega_{n} \dot{x}+\omega_{n}^{2}\left(1+k_{0} \sin (\omega t)\right) f(x)=\omega_{n}^{2} F \\
& +\omega_{n}^{2} F_{p} \cos \left(\omega_{p} t\right)+\omega_{n}^{2} F_{g} \cos \left(\omega_{g} t\right)+\omega^{2} E \cos (\omega t)
\end{aligned}
$$

By defining the following dimensionless parameters

$$
\begin{gathered}
\hat{t}=\omega_{n} t \\
u=\frac{x}{b}, \quad u_{p}=\frac{x_{p}}{b}, \quad u_{g}=\frac{x_{g}}{b} \\
\Omega=\frac{\omega}{\omega_{n}}, \quad \Omega_{p}=\frac{\omega_{p}}{\omega_{n}}, \quad \Omega_{g}=\frac{\omega_{g}}{\omega_{n}}
\end{gathered}
$$

Equations (6),(7) and (15) are written in the following dimensionless form. 


$$
\begin{aligned}
& m_{p} \hat{\ddot{u}}_{p}+2 \zeta_{p} \hat{\dot{u}}_{p}+2 \zeta \hat{\dot{u}}+k_{p} u_{p}+\alpha_{p} u_{p}^{3}+\left(1+k_{o} \cos (\Omega \hat{t})\right) f(u)=0 \\
& m_{g} \hat{\ddot{u}}_{g}+2 \zeta_{g} \hat{\dot{u}}_{g}-2 \zeta \hat{\dot{u}}+k_{g} u_{g}+\alpha_{g} u_{g}^{3}-\left(1+k_{o} \cos (\Omega \hat{t})\right) f(u)=0 \\
& -\hat{\ddot{u}}_{p}+\hat{\ddot{u}}_{g}+\hat{\ddot{u}}+2 \zeta \hat{\dot{u}}+\left(1+k_{o} \cos (\Omega \hat{t})\right) f(u) \\
& \quad=F+F_{p} \cos \left(\Omega_{p} \hat{t}\right)+F_{g} \cos \left(\Omega_{g} \hat{t}\right)+\Omega^{2} E \cos (\Omega \hat{t})
\end{aligned}
$$

where the ratio of the parameter corresponded to the suspension system over the parameter corresponded to the gear pairs in mesh are expressed as follow.

$$
\begin{gathered}
m_{p}=\frac{M_{p}}{M}, \quad m_{g}=\frac{M_{g}}{M}, \\
k_{p}=\frac{K_{p}}{K}, \quad \alpha_{p}=\frac{b^{2} K_{p o}}{K}, \quad k_{g}=\frac{K_{g}}{K}, \quad \alpha_{g}=\frac{b^{2} K_{g o}}{K} \\
\zeta_{p}=\frac{C_{p}}{2 \sqrt{K M}}, \quad \zeta_{g}=\frac{C_{g}}{2 \sqrt{K M}}
\end{gathered}
$$

Finally, one can write Eqs. (17)-(19) in the following matrix form.

$$
\begin{aligned}
& {\left[\begin{array}{ccc}
m_{p} & 0 & 0 \\
0 & m_{g} & 0 \\
-1 & 1 & 1
\end{array}\right]\left[\begin{array}{c}
\hat{\ddot{u}}_{p} \\
\hat{\ddot{u}}_{g} \\
\hat{\ddot{u}}
\end{array}\right]+2\left[\begin{array}{ccc}
\zeta_{p} & 0 & \zeta \\
0 & \zeta_{g} & -\zeta \\
0 & 0 & \zeta
\end{array}\right]\left[\begin{array}{c}
\hat{\dot{u}}_{p} \\
\hat{\dot{u}}_{g} \\
\hat{\dot{u}}^{\prime}
\end{array}\right]} \\
& +\left[\begin{array}{ccc}
k_{p} & 0 & +\left(1+k_{0} \cos (\Omega \hat{t})\right) \\
0 & k_{g} & -\left(1+k_{0} \cos (\Omega \hat{t})\right) \\
0 & 0 & +\left(1+k_{0} \cos (\Omega \hat{t})\right)
\end{array}\right]\left[\begin{array}{c}
u_{p} \\
u_{g} \\
f(u)
\end{array}\right]+\left[\begin{array}{ccc}
\alpha_{p} & 0 & 0 \\
0 & \alpha_{g} & 0 \\
0 & 0 & 0
\end{array}\right]\left[\begin{array}{c}
u_{p}^{3} \\
u_{g}^{3} \\
u^{3}
\end{array}\right] \\
& =\left[\begin{array}{c}
0 \\
0 \\
F+F_{p} \cos \left(\Omega_{p} \hat{t}\right)+F_{g} \cos \left(\Omega_{g} \hat{t}\right)+\Omega^{2} E \cos (\Omega \hat{t})
\end{array}\right]
\end{aligned}
$$

with the following dimensionless backlash function

$$
f(u)=\left\{\begin{array}{cc}
u-1 & +1<u \\
0 & -1 \leq u \leq+1 \\
u+1 & u<-1
\end{array}\right.
$$

Equation (21) is a damped parametrically and externally excited nonlinear system of coupled equations and the non-symmetric nature of the system is due to the choice of the coordinates. 


\section{Analytical Calculations}

The goal of this section is to use Poincare-Lindstedt method to study the stability and bifurcation of the equilibria around the unstable tongues. This is done under assumption that the system operates under constant velocity and relatively high load. For a symmetric system, where both driving and driven shafts have the same parameters we have

$$
\begin{gathered}
m=m_{p}=m_{g} \\
k=k_{p}=k_{g} \\
\alpha=\alpha_{p}=\alpha_{g}
\end{gathered}
$$

By imposing the permanent contact condition, Eq. (21) become a system of damped nonlinear differential equations with periodic time-varying coefficients.

$$
\begin{aligned}
& {\left[\begin{array}{rrr}
m & 0 & 0 \\
0 & m & 0 \\
-1 & 1 & 1
\end{array}\right]\left[\begin{array}{l}
\hat{\ddot{u}}_{p} \\
\hat{\ddot{u}}_{g} \\
\hat{\ddot{u}}
\end{array}\right]+2\left[\begin{array}{ccc}
\zeta_{p} & 0 & \zeta \\
0 & \zeta_{g} & -\zeta \\
0 & 0 & \zeta
\end{array}\right]\left[\begin{array}{c}
\hat{\dot{u}}_{p} \\
\hat{\dot{u}}_{g} \\
\hat{\dot{u}}
\end{array}\right]} \\
& +\left[\begin{array}{lll}
k & 0 & +\left(1+k_{0} \cos (\Omega \hat{t})\right) \\
0 & k & -\left(1+k_{0} \cos (\Omega \hat{t})\right) \\
0 & 0 & +\left(1+k_{0} \cos (\Omega \hat{t})\right)
\end{array}\right]\left[\begin{array}{l}
u_{p} \\
u_{g} \\
u
\end{array}\right]+\left[\begin{array}{lll}
\alpha & 0 & 0 \\
0 & \alpha & 0 \\
0 & 0 & 0
\end{array}\right]\left[\begin{array}{l}
u_{p}^{3} \\
u_{g}^{3} \\
u^{3}
\end{array}\right] \\
& =\left[\begin{array}{l}
0 \\
F+F_{p} \cos (\Omega \hat{t})+F_{g} \cos \left(\Omega_{g} \hat{t}\right)+\Omega^{2} E \cos (\Omega \hat{t})
\end{array}\right] \\
& +\left[\begin{array}{l}
+\left(1+k_{0} \cos (\Omega \hat{t})\right) \\
-\left(1+k_{0} \cos (\Omega \hat{t})\right) \\
+\left(1+k_{0} \cos (\Omega \hat{t})\right)
\end{array}\right]
\end{aligned}
$$

To study the stability of the corresponding undamped homogenous system, the time varying and nonlinear terms are perturbed as follow.

$$
\left[\begin{array}{rrr}
m & 0 & 0 \\
0 & m & 0 \\
-1 & 1 & 1
\end{array}\right]\left[\begin{array}{c}
\hat{\ddot{u}}_{p} \\
\hat{\ddot{u}}_{g} \\
\hat{\ddot{u}}
\end{array}\right]+\left[\begin{array}{cc}
k & 0+(1+\epsilon \cos (\Omega \hat{t})) \\
0 & k-(1+\epsilon \cos (\Omega \hat{t})) \\
0 & 0+(1+\epsilon \cos (\Omega \hat{t}))
\end{array}\right]\left[\begin{array}{l}
u_{p} \\
u_{g} \\
u
\end{array}\right]+\epsilon\left[\begin{array}{lll}
\alpha & 0 & 0 \\
0 & \alpha & 0 \\
0 & 0 & 0
\end{array}\right]\left[\begin{array}{c}
u_{p}^{3} \\
u_{g}^{3} \\
u^{3}
\end{array}\right]=0
$$


where $\epsilon$ is a small parameter and the following transformation of variable is used to implement Poincare-Lindstedt method.

$$
\tau=\Omega \hat{t}, \quad u_{p}^{\prime}=\frac{d u_{p}}{d \tau}, \quad u_{g}^{\prime}=\frac{d u_{g}}{d \tau}, \quad u^{\prime}=\frac{d u}{d \tau}
$$

which results in the following system of equations.

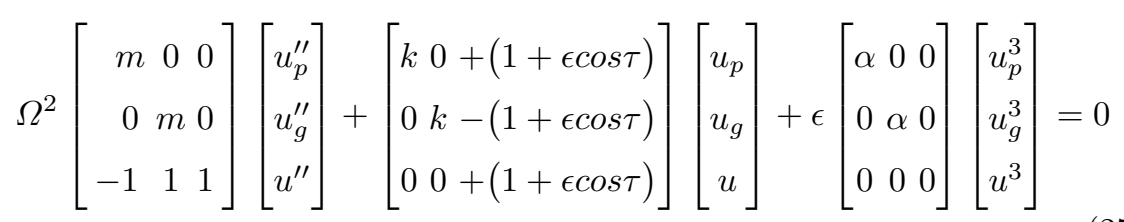

where prime represent differentiation with respect to the new variable $\tau$. Now by expanding the variables in the following power series

$$
\begin{aligned}
& u_{p}=u_{0 p}+u_{1 p} \epsilon+u_{2 p} \epsilon^{2}+u_{3 p} \epsilon^{3}+\ldots \\
& u_{g}=u_{0 g}+u_{1 g} \epsilon+u_{2 g} \epsilon^{2}+u_{3 g} \epsilon^{3}+\ldots \\
& u=u_{0}+u_{1} \epsilon+u_{2} \epsilon^{2}+u_{3} \epsilon^{3}+\ldots \\
& \Omega=\Omega_{0}+\Omega_{1} \epsilon+\Omega_{2} \epsilon^{2}+\Omega_{3} \epsilon^{3}+\ldots
\end{aligned}
$$

substituting Eqs. (28)-(31) in Eq. (27), neglecting terms of $O\left(\epsilon^{2}\right)$, and collecting terms of the same power the following system of equations are obtained.

$$
\begin{aligned}
& \Omega_{0}^{2}\left[\begin{array}{ccc}
m & 0 & 0 \\
0 & m & 0 \\
-1 & 1 & 1
\end{array}\right]\left[\begin{array}{l}
u_{0 p}^{\prime \prime} \\
u_{0 g}^{\prime \prime} \\
u_{0}^{\prime \prime}
\end{array}\right]+\left[\begin{array}{rrr}
k & 0 & 1 \\
0 & k & -1 \\
0 & 0 & 1
\end{array}\right]\left[\begin{array}{l}
u_{0 p} \\
u_{0 g} \\
u_{0}
\end{array}\right]=0 \\
& \Omega_{0}^{2}\left[\begin{array}{rrr}
m & 0 & 0 \\
0 & m & 0 \\
-1 & 1 & 1
\end{array}\right]\left[\begin{array}{l}
u_{1 p}^{\prime \prime} \\
u_{1 g}^{\prime \prime} \\
u_{1}^{\prime \prime}
\end{array}\right]+\left[\begin{array}{lll}
k & 0 & 1 \\
0 & k & -1 \\
0 & 0 & 1
\end{array}\right]\left[\begin{array}{l}
u_{1 p} \\
u_{1 g} \\
u_{1}
\end{array}\right] \\
& =-2 \Omega_{0} \Omega_{1}\left[\begin{array}{ccc}
m & 0 & 0 \\
0 & m & 0 \\
-1 & 1 & 1
\end{array}\right]\left[\begin{array}{l}
u_{0 p}^{\prime \prime} \\
u_{0 g}^{\prime \prime} \\
u_{0}^{\prime \prime}
\end{array}\right] \\
& -\left[\begin{array}{r}
1 \\
-1 \\
1
\end{array}\right] u_{0} \cos \tau-\left[\begin{array}{lll}
\alpha & 0 & 0 \\
0 & \alpha & 0 \\
0 & 0 & 0
\end{array}\right]\left[\begin{array}{l}
u_{p}^{3} \\
u_{g}^{3} \\
u^{3}
\end{array}\right]
\end{aligned}
$$

Equation (32) is a linear homogenous system of equations with following solution.

$$
\left[\begin{array}{l}
u_{0 p} \\
u_{0 g} \\
u_{0}
\end{array}\right]=\left[\begin{array}{l}
A \\
B \\
C
\end{array}\right] e^{\lambda \tau}
$$


By substituting Eq. (34) in Eq. (32), the following equation is obtained

$$
\left[\begin{array}{ccc}
m \Omega_{0}^{2} \lambda^{2}+k & 0 & +1 \\
0 & m \Omega_{0}^{2} \lambda^{2}+k & -1 \\
-\Omega_{0}^{2} \lambda^{2} & +\Omega_{0}^{2} \lambda^{2} & -\Omega_{0}^{2} \lambda^{2}+1
\end{array}\right]\left[\begin{array}{l}
A \\
B \\
C
\end{array}\right] e^{\lambda \tau}=0
$$

which result in the corresponding eigenvalues and eigenvectors of the system.

$$
\begin{gathered}
\lambda_{1}^{2}=\frac{k}{m \Omega_{0}^{2}} i^{2}, \quad \lambda_{2}^{2}=\frac{2 k-a}{2 m \Omega_{0}^{2}} i^{2}, \quad \lambda_{3}^{2}=\frac{2 k-b}{2 m \Omega_{0}^{2}} i^{2} \\
v_{1}=\left[\begin{array}{l}
1 \\
1 \\
0
\end{array}\right], v_{2}=\left[\begin{array}{c}
-2 \\
+2 \\
a
\end{array}\right], v_{3}=\left[\begin{array}{c}
-2 \\
+2 \\
b
\end{array}\right]
\end{gathered}
$$

where constants $a$ and $b$ are a function of system parameters $m$ and $k$, and $i$ represents the imaginary unit, satisfying $i^{2}=-1$.

$$
\begin{aligned}
& a=-(m-k+2)+\sqrt{(m+k+2)^{2}-4 k m} \\
& b=-(m-k+2)-\sqrt{(m+k+2)^{2}-4 k m}
\end{aligned}
$$

And the following relationships holds between $a$ and $b$.

$$
a b=-8 k
$$

The nonsymmetric nature of Eq. (32) suggests that the eigenvectors of this system do not form an orthogonal subspace and consequently according to the spectral Theorem Eq. (32) cannot be diagonalized. Specifically, because the second and third eigenvectors are not orthogonal. But as it is shown below, for the special case of $k=1$, the corresponding $M^{-1} K$ matrix is symmetric, the second and third eigenvectors are orthogonal and the system is diagonalizable. However in this work, the general form of Eq. (32) is solved for every $k$.

$$
v_{1} v_{2}=0, v_{1} v_{3}=0, v_{2} v_{3}=8(1-k)
$$

For positive values of $k$ and $m$, the following condition is always satisfied, therefore the values of $a$ and $b$ and consequently all the eigenvectors are real.

$$
(m+k+2)^{2}-4 k m>0
$$

Besides, since the following equation are positive for any value of $k$ and $m$.

$$
\begin{aligned}
& 2 k-a=(m+k+2)-\sqrt{(m+k+2)^{2}-4 k m} \\
& 2 k-b=(m+k+2)+\sqrt{(m+k+2)^{2}-4 k m}
\end{aligned}
$$


the three eigenvalues expressed by Eq. (36) are always complex. Therefore, for any value of the parameters, Eq. (32) has the following three natural frequencies.

$$
\omega_{n 1}^{2}=\frac{k}{m \Omega_{0}^{2}}, \quad \omega_{n 2}^{2}=\frac{2 k-a}{2 m \Omega_{0}^{2}}, \quad \omega_{n 3}^{2}=\frac{2 k-b}{2 m \Omega_{0}^{2}}
$$

such that the following relationship holds between them.

$$
\begin{gathered}
\omega_{n 2}^{2} \omega_{n 3}^{2}=\frac{k}{m \Omega_{0}^{4}} \\
\omega_{n 1}^{2}=\Omega_{0}^{2} \omega_{n 2}^{2} \omega_{n 3}^{2}
\end{gathered}
$$

By having the eigenvalues and eigenvectors of Eq. (32), one can write the solution of this equation in the following form.

$$
\begin{array}{r}
{\left[\begin{array}{c}
u_{0 p} \\
u_{0 g} \\
u_{0}
\end{array}\right]=\left[\begin{array}{l}
1 \\
1 \\
0
\end{array}\right]\left(A_{1} \cos \left(\omega_{n 1} \tau\right)+A_{2} \sin \left(\omega_{n 1} \tau\right)\right)} \\
+\left[\begin{array}{c}
-2 \\
+2 \\
a
\end{array}\right]\left(B_{1} \cos \left(\omega_{n 2} \tau\right)+B_{2} \sin \left(\omega_{n 2} \tau\right)\right) \\
+\left[\begin{array}{c}
-2 \\
+2 \\
b
\end{array}\right]\left(C_{1} \cos \left(\omega_{n 3} \tau\right)+C_{2} \sin \left(\omega_{n 3} \tau\right)\right)
\end{array}
$$

Substituting Eq. (47) in Eq. (33) results in the following equation. 


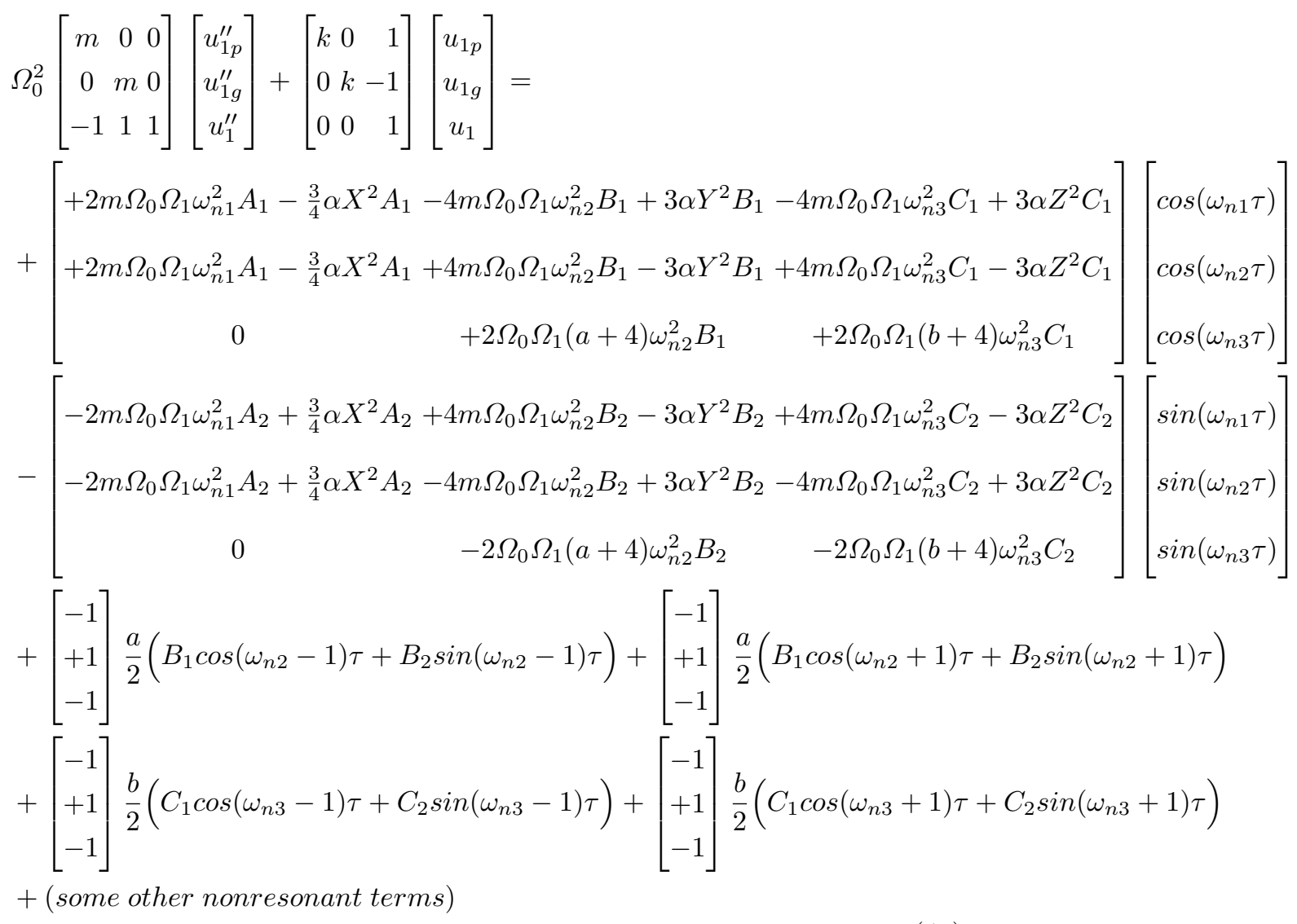

Equation (48) contains harmonic functions with frequencies equal to

$$
\omega_{n 1}, \omega_{n 2}, \omega_{n 3}, \omega_{n 2} \pm 1, \omega_{n 3} \pm 1, \ldots
$$

and parameters $X, Y$ and $Z$ are defined as follow.

$$
\begin{aligned}
& X^{2}=\left(A_{1}^{2}+A_{2}^{2}\right)+8\left(B_{1}^{2}+B_{2}^{2}\right)+8\left(C_{1}^{2}+C_{2}^{2}\right) \\
& Y^{2}=\left(A_{1}^{2}+A_{2}^{2}\right)+2\left(B_{1}^{2}+B_{2}^{2}\right)+4\left(C_{1}^{2}+C_{2}^{2}\right) \\
& Z^{2}=\left(A_{1}^{2}+A_{2}^{2}\right)+4\left(B_{1}^{2}+B_{2}^{2}\right)+2\left(C_{1}^{2}+C_{2}^{2}\right)
\end{aligned}
$$

3.1 Primary Parametric Resonance Due to the Second Natural Frequency

In the general case, all the harmonic terms with frequencies equal to the three natural frequencies in Eq. (48) are secular and must be removed. But for 
the following special case of the second natural frequency more terms become secular [10].

$$
\omega_{n 2}-1=-\omega_{n 2} \rightarrow \omega_{n 2}=\frac{1}{2}
$$

Imposing this condition to Eq. (44) gives the emanating frequency of the first tongue related the second natural frequency.

$$
\Omega_{0}^{2}=\frac{2(2 k-a)}{m}
$$

Inserting Eq. (52) into Eqs. (45) and (46) provides the other two natural frequencies.

$$
\omega_{n 1}^{2}=\frac{k}{2(2 k-a)}, \omega_{n 2}^{2}=\frac{1}{4}, \omega_{n 3}^{2}=\frac{k m}{(2 k-a)^{2}}
$$

By substituting Eq. (53) in Eq. (48), the following matrix form of the secular terms is obtained.

$$
\left[\begin{array}{r}
+2 m \Omega_{0} \Omega_{1} \omega_{n 1}^{2} A_{1}-\frac{3}{4} \alpha X^{2} A_{1}-4 m \Omega_{0} \Omega_{1} \omega_{n 2}^{2} B_{1}+3 \alpha Y^{2} B_{1}-\frac{a}{2} B_{1}-4 m \Omega_{0} \Omega_{1} \omega_{n 3}^{2} C_{1}+3 \alpha Z^{2} C_{1} \\
+2 m \Omega_{0} \Omega_{1} \omega_{n 1}^{2} A_{1}-\frac{3}{4} \alpha X^{2} A_{1}+4 m \Omega_{0} \Omega_{1} \omega_{n 2}^{2} B_{1}-3 \alpha Y^{2} B_{1}+\frac{a}{2} B_{1}+4 m \Omega_{0} \Omega_{1} \omega_{n 3}^{2} C_{1}-3 \alpha Z^{2} C_{1} \\
+2 \Omega_{0} \Omega_{1}(a+4) \omega_{n 2}^{2} B_{1}-\frac{a}{2} B_{1} \\
0
\end{array}\right]
$$

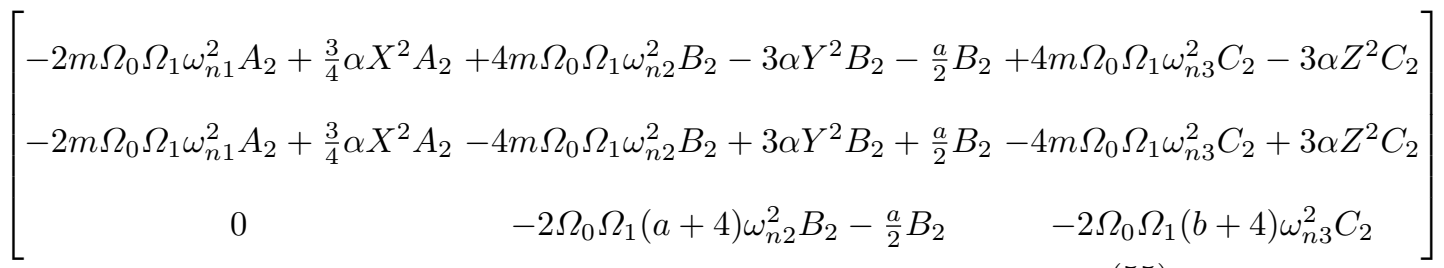

Imposing the zero determinant condition to these two matrices, where $\alpha=0$, provide the two first-order multipliers of Eq. (31) for the transition curves of the unstable tongue. Such that the negative and positive signs are related to sine and cosine multipliers associated to the left and right transition curves, respectively.

$$
\Omega_{1}= \pm \frac{a(b+2 m+4)}{2 m \Omega_{0}(a-b)}
$$

Besides, the newborn nontrivial equilibria exist if and only if one of the columns of Eqs. (54) and (55) is in the column space of Eq. (35). So the Cramer's rule can be used as follow to impose the solvability condition ([10] page 399). 


$$
\left[\begin{array}{ccc}
a_{1 j} & 0 & +1 \\
a_{2 j} & m \Omega_{0}^{2} \lambda^{2}+k & -1 \\
a_{3 j} & +\Omega_{0}^{2} \lambda^{2} & -\Omega_{0}^{2} \lambda^{2}+1
\end{array}\right]=0,\left[\begin{array}{ccc}
m \Omega_{0}^{2} \lambda^{2}+k & a_{1 j} & +1 \\
0 & a_{2 j} & -1 \\
-\Omega_{0}^{2} \lambda^{2} & a_{3 j} & -\Omega_{0}^{2} \lambda^{2}+1
\end{array}\right]=0,\left[\begin{array}{ccr}
m \Omega_{0}^{2} \lambda^{2}+k & 0 & a_{1 j} \\
0 & m \Omega_{0}^{2} \lambda^{2}+k & a_{2 j} \\
-\Omega_{0}^{2} \lambda^{2} & +\Omega_{0}^{2} \lambda^{2} & a_{3 j}
\end{array}\right]=0
$$

But only the second columns in Eqs. (54) and (55) satisfy Eq. (57) for nontrivial $B_{1}$ and $B_{2}$,which results in the following two equations.

$$
\begin{aligned}
& \left(-4 m \Omega_{0} \Omega_{1} \omega_{n 2}^{2} B_{2}+3 \alpha Y^{2} B_{2}+\frac{a}{2} B_{2}\right)+\left(\frac{a}{4 \Omega_{0}^{2} \omega_{n 2}^{2}}\right)\left(-2 \Omega_{0} \Omega_{1}(a+4) \omega_{n 2}^{2} B_{2}-\frac{a}{2} B_{2}\right)=0 \\
& \left(+4 m \Omega_{0} \Omega_{1} \omega_{n 2}^{2} B_{1}-3 \alpha Y^{2} B_{1}+\frac{a}{2} B_{1}\right)+\left(\frac{a}{4 \Omega_{0}^{2} \omega_{n 2}^{2}}\right)\left(+2 \Omega_{0} \Omega_{1}(a+4) \omega_{n 2}^{2} B_{1}-\frac{a}{2} B_{1}\right)=0
\end{aligned}
$$

Equations (58) and (59) can be simplified as follow by using the relationship between parameters $a, b$ and the second natural frequency.

$$
\begin{aligned}
& \left(+6 \alpha\left(B_{1}^{2}+B_{2}^{2}\right)-\frac{a(a-b)}{2 \Omega_{0}}\left(\Omega_{1}+\frac{a(b+2 m+4)}{2 m \Omega_{0}(a-b)}\right)\right) B_{2}=0 \\
& \left(-6 \alpha\left(B_{1}^{2}+B_{2}^{2}\right)+\frac{a(a-b)}{2 \Omega_{0}}\left(\Omega_{1}-\frac{a(b+2 m+4)}{2 m \Omega_{0}(a-b)}\right)\right) B_{1}=0
\end{aligned}
$$

By defining the polar coordinates, $B_{1}=R_{B} \cos \theta_{B}$ and $B_{2}=R_{B} \sin \theta_{B}$, the alternate polar form of Eqs. (60) and (61) are obtained.

$$
\begin{aligned}
& \left(+6 \alpha R_{B}^{2}-\frac{a(a-b)}{2 \Omega_{0}}\left(\Omega_{1}+\frac{a(b+2 m+4)}{2 m \Omega_{0}(a-b)}\right)\right) R_{B} \sin \theta_{B}=0 \\
& \left(-6 \alpha R_{B}^{2}+\frac{a(a-b)}{2 \Omega_{0}}\left(\Omega_{1}-\frac{a(b+2 m+4)}{2 m \Omega_{0}(a-b)}\right)\right) R_{B} \cos \theta_{B}=0
\end{aligned}
$$

Solving (62) and (63) results in the following five equilibrium points.

$$
\begin{array}{ll}
R_{B 1}=0 & \\
\left.R_{B 2,3}^{2}=\frac{a(a-b)}{12 \alpha \Omega_{0}}\left(\Omega_{1}+\frac{a(b+2 m+4)}{2 m \Omega_{0}(a-b)}\right)\right) & \theta_{B}=\frac{\pi}{2}, \frac{3 \pi}{2} \\
\left.R_{B 4,5}^{2}=\frac{a(a-b)}{12 \alpha \Omega_{0}}\left(\Omega_{1}-\frac{a(b+2 m+4)}{2 m \Omega_{0}(a-b)}\right)\right) & \theta_{B}=0, \pi
\end{array}
$$

Such that $R_{1}$ is corresponded to the trivial equilibria at origin and each of $R_{2,3}$ and $R_{4,5}$ is corresponded to two nontrivial equilibria located $\pi(\mathrm{rad})$ apart from each other. The local stability of these equilibrium points is determined by the eigenvalues of the Jacobian matrix corresponded to Eqs. (60) and (61) [9]. 
$J=\left[\begin{array}{cc}+12 \alpha B_{1} B_{2} & +6 \alpha\left(B_{1}^{2}+3 B_{2}^{2}\right)-\frac{a(a-b)}{2 \Omega_{0}}\left(\Omega_{1}+\frac{a(b+2 m+4)}{2 m \Omega_{0}(a-b)}\right) \\ -6 \alpha\left(3 B_{1}^{2}+B_{2}^{2}\right)+\frac{a(a-b)}{2 \Omega_{0}}\left(\Omega_{1}-\frac{a(b+2 m+4)}{2 m \Omega_{0}(a-b)}\right) & -12 \alpha B_{1} B_{2}\end{array}\right]$

Alternatively, for a $2 \times 2$ matrix the Trace $(T r)$ and Determinant $(D e t)$ of the Jacobian matrix can be used to form the characteristic equation.

$$
\lambda^{2}-\operatorname{Tr}(J) \lambda+\operatorname{Det}(J)=0
$$

The $\operatorname{Tr}$ of Eq. (67) is always equal to zero and the value of the $D e t$ is expressed by the following equation.

$$
\begin{aligned}
\text { Det }=108 \alpha^{2}\left(B_{1}^{2}+B_{2}^{2}\right)^{2} & -6 \alpha \frac{a(a-b)}{2 \Omega_{0}}\left(4 \Omega_{1}\left(B_{1}^{2}+B_{2}^{2}\right)+\frac{a(b+2 m+4)}{m \Omega_{0}(a-b)}\left(B_{1}^{2}-B_{2}^{2}\right)\right) \\
& +\frac{a^{2}(a-b)^{2}}{4 \Omega_{0}^{2}}\left(\Omega_{1}^{2}-\frac{a^{2}(b+2 m+4)^{2}}{4 m^{2} \Omega_{0}^{2}(a-b)^{2}}\right)
\end{aligned}
$$

Since the value of $\operatorname{Tr}$ is always equal to zero, for every $\Omega_{1}$ the eigenvalues of the Jacobian matrix have the same magnitude with opposite signs. Such that for the positive values of the Det both eigenvalues are on the imaginary axis and the equilibria is stable and for the negative values of the Det both eigenvalues are on the real axis and the equilibria is unstable. Recall that a pitchfork bifurcation generically occurs when the determinant of the Jacobean matrix become zero [12]. Transforming Eq. (69) into the polar coordinate and substituting Eqs. (64)-(66) in it yields the following expression for Det at the equilibrium points.

$$
\begin{aligned}
& \text { Det }=+\frac{a^{2}(a-b)^{2}}{4 \Omega_{0}^{2}}\left(\Omega_{1}^{2}-\frac{a^{2}(b+2 m+4)^{2}}{4 m^{2} \Omega_{0}^{2}(a-b)^{2}}\right) \\
& \text { Det }=+\frac{a^{3}(b+2 m+4)(a-b)}{2 m \Omega_{0}^{3}}\left(\Omega_{1}+\frac{a(b+2 m+4)}{2 m \Omega_{0}(a-b)}\right) \\
& \text { Det }=-\frac{a^{3}(b+2 m+4)(a-b)}{2 m \Omega_{0}^{3}}\left(\Omega_{1}-\frac{a(b+2 m+4)}{2 m \Omega_{0}(a-b)}\right)
\end{aligned}
$$

The Det of the equilibrium points for different values of $\Omega_{1}$ are demonstrated in Fig. 2. 


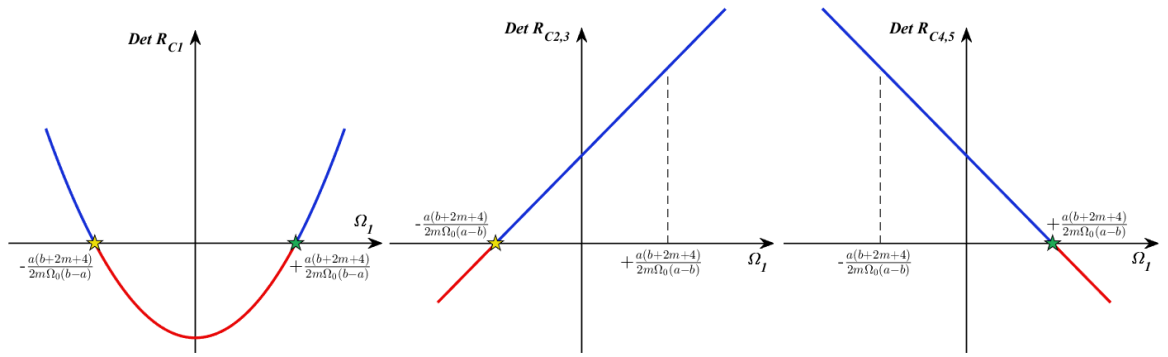

Fig. 2 Determinant at the equilibrium points around the resonance tongue due to the second natural frequency

Besides, Eqs. (65) and (66) provide the square value of the equilibrium points which impose a condition on the value of $\Omega_{1}$. Therefore, existence of the real equilibria requires that for the case of hardening cubic nonlinearity $(\alpha>0)$.

$$
\begin{array}{llll}
R_{B 1} & \text { Exist } & \forall & \omega_{1} \\
R_{B 2,3} & \text { Exist } & \forall & \omega_{1}>-\frac{a(b+2 m+4)}{2 m \Omega_{0}(a-b)} \\
R_{B 4,5} & \text { Exist } & \forall & \omega_{1}>+\frac{a(b+2 m+4)}{2 m \Omega_{0}(a-b)}
\end{array}
$$

and for the case of softening cubic nonlinearity $(\alpha<0)$

$$
\begin{array}{lllll}
R_{B 1} & \text { Exist } & \forall & \omega_{1} \\
R_{B 2,3} & \text { Exist } & \forall & \omega_{1}<-\frac{a(b+2 m+4)}{2 m \Omega_{0}(a-b)} \\
R_{B 4,5} & \text { Exist } & \forall & \omega_{1}<+\frac{a(b+2 m+4)}{2 m \Omega_{0}(a-b)}
\end{array}
$$

The corresponding bifurcation diagram for hardening and softening cases are plotted in Fig. 3. 


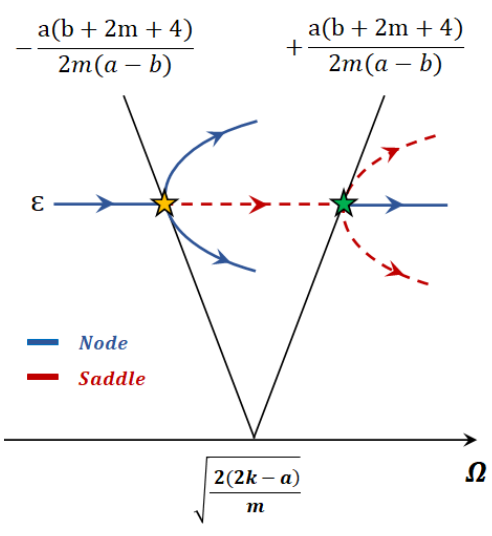

Hardening nonlinearity

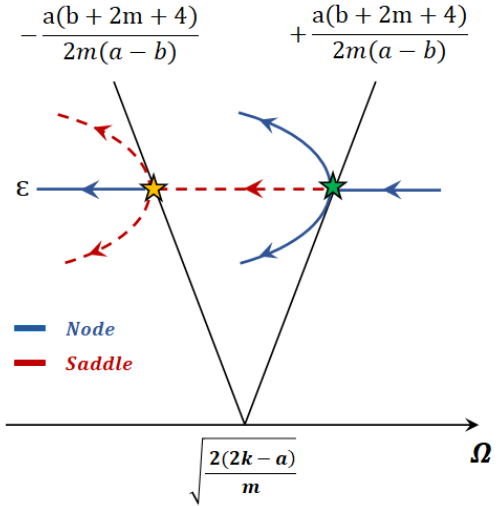

Softening nonlinearity

Fig. 3 Subcritical and supercritical pitchfork bifurcations around the resonance tongue due to the second natural frequenc

This analysis shows that for both hardening and softening nonlinearities, as one crosses the transition curves (for the constant value of $\epsilon$ ) subcritical and supercritical pitchfork bifurcations (birth of new equilibria) occur. As illustrated in Fig. 3, for the hardening nonlinearity, Eqs. (73)-(75) requires that the origin is the only equilibria at the left-hand side of the unstable tongue. Meanwhile, Fig. 2 shows that by quasi statically increasing the value of $\Omega_{1}$ and approaching the left transition curve, Det of both $R_{B 1}$ and $R_{B 2,3}$ merge to zero. Consequently, by crossing the left transition curve Det of the Jacobian matrix at $R_{B 1}$ and $R_{B 2,3}$ cross $\Omega_{1}$ axis and a supercritical pitchfork bifurcation occurs, such that the equilibria at the origin becomes unstable and two new stable equilibria are born. Figure 2 shows that as $\Omega_{1}$ increase more and crosses the right transition curve, Det of Jacobian matrix at $R_{B 1}$ and $R_{B 4,5}$ cross the $\Omega_{1}$ axis and a subcritical pitchfork bifurcation occurs where the origin become stable again and two new unstable equilibria are born. Figure 3 shows that the same sequence of events happens for the case of softening nonlinearity. The only difference is that Eqs. (76)-(78) requires that origin is the only equilibria on the right-hand side of the unstable tongue and by quasi statically decreasing $\Omega_{1}$ and approaching the right transition curve, Det of $R_{B 1}$ and $R_{B 4,5}$ merge to zero and cause a supercritical pitchfork bifurcation. Greater decrease in the value of $\Omega_{1}$ causes a subcritical pitchfork bifurcation where the left transition curve is crossed and $D e t$ of $R_{B 1}$ and $R_{B 2,3}$ approach zero and cross the $\Omega_{1}$ axis. 
3.2 Primary Parametric Resonance Due to the Third Natural Frequency

Alternatively, for the following special case of the third natural frequency other terms in Eq. (48) become secular.

$$
\omega_{n 3}-1=-\omega_{n 3} \rightarrow \omega_{n 3}=\frac{1}{2}
$$

Imposing this condition to Eq. (44) gives the emanating frequency of the unstable tongue related the third natural frequency.

$$
\Omega_{0}^{2}=\frac{2(2 k-b)}{m}
$$

Inserting Eq. (80) into Eqs. (45) and (46) provides the other two natural frequencies.

$$
\omega_{n 1}^{2}=\frac{k}{2(2 k-b)}, \omega_{n 2}^{2}=\frac{k m}{(2 k-b)^{2}}, \omega_{n 3}^{2}=\frac{1}{4}
$$

By substituting these three natural frequencies in Eq. (48), the following matrix form of the secular terms is obtained.

$$
\begin{aligned}
& {\left[\begin{array}{r}
+2 m \Omega_{0} \Omega_{1} \omega_{n 1}^{2} A_{1}-\frac{3}{4} \alpha X^{2} A_{1}-4 m \Omega_{0} \Omega_{1} \omega_{n 2}^{2} B_{1}+3 \alpha Y^{2} B_{1}-4 m \Omega_{0} \Omega_{1} \omega_{n 3}^{2} C_{1}+3 \alpha Z^{2} C_{1}-\frac{b}{2} C_{1} \\
+2 m \Omega_{0} \Omega_{1} \omega_{n 1}^{2} A_{1}-\frac{3}{4} \alpha X^{2} A_{1}+4 m \Omega_{0} \Omega_{1} \omega_{n 2}^{2} B_{1}-3 \alpha Y^{2} B_{1}+4 m \Omega_{0} \Omega_{1} \omega_{n 3}^{2} C_{1}-3 \alpha Z^{2} C_{1}+\frac{b}{2} C_{1} \\
0
\end{array} \begin{array}{rr}
+2 \Omega_{0} \Omega_{1}(a+4) \omega_{n 2}^{2} B_{1} & +2 \Omega_{0} \Omega_{1}(b+4) \omega_{n 3}^{2} C_{1}-\frac{b}{2} C_{1}
\end{array}\right]}
\end{aligned}
$$

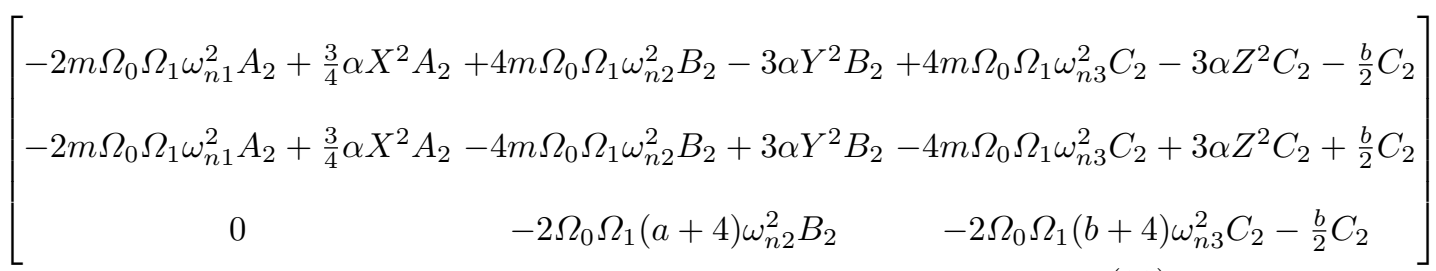

Similarly, imposing the zero determinant condition to Eqs, (82) and (83) where $\alpha=0$, provides the two first-order multipliers of Eq. (31) for the transition curves of the unstable tongue. Such that the negative and positive signs are related to sine and cosine multipliers associated to the left and right transition curves, respectively.

$$
\Omega_{1}= \pm \frac{b(a+2 m+4)}{2 m \Omega_{0}(b-a)}
$$


In this case, only the third columns in Eqs. (82) and (83) is in the column space of Eq. (35) and satisfy Eq. (57) for non-trivial $C_{1}$ and $C_{2}$, which results in the following two equations.

$$
\left(-4 m \Omega_{0} \Omega_{1} \omega_{n 3}^{2} C_{2}+3 \alpha Z^{2} C_{2}+\frac{b}{2} C_{2}\right)+\left(\frac{b}{4 \Omega_{0}^{2} \omega_{n 3}^{2}}\right)\left(-2 \Omega_{0} \Omega_{1}(b+4) \omega_{n 3}^{2} C_{2}-\frac{b}{2} C_{2}\right)=0
$$

$\left(+4 m \Omega_{0} \Omega_{1} \omega_{n 3}^{2} C_{1}-3 \alpha Z^{2} C_{1}+\frac{b}{2} C_{1}\right)+\left(\frac{b}{4 \Omega_{0}^{2} \omega_{n 3}^{2}}\right)\left(+2 \Omega_{0} \Omega_{1}(b+4) \omega_{n 3}^{2} C_{1}-\frac{b}{2} C_{1}\right)=0$

Equations (85) and (86) can be simplified as follow by using the relationship between parameters $a, b$ and the third natural frequency.

$$
\begin{aligned}
& \left(+6 \alpha\left(C_{1}^{2}+C_{2}^{2}\right)-\frac{b(b-a)}{2 \Omega_{0}}\left(\Omega_{1}+\frac{b(a+2 m+4)}{2 m \Omega_{0}(b-a)}\right)\right) C_{2}=0 \\
& \left(-6 \alpha\left(C_{1}^{2}+C_{2}^{2}\right)+\frac{b(b-a)}{2 \Omega_{0}}\left(\Omega_{1}-\frac{b(a+2 m+4)}{2 m \Omega_{0}(b-a)}\right)\right) C_{1}=0
\end{aligned}
$$

By defining the polar coordinates, $C_{1}=R_{C} \cos \theta_{C}$ and $C_{2}=R_{C} \sin \theta_{C}$, the alternate polar form of (87) and (88) are obtained.

$$
\begin{aligned}
& \left(+6 \alpha R_{C}^{2}-\frac{b(b-a)}{2 \Omega_{0}}\left(\Omega_{1}+\frac{b(a+2 m+4)}{2 m \Omega_{0}(b-a)}\right)\right) R_{C} \sin \theta_{C}=0 \\
& \left(-6 \alpha R_{C}^{2}+\frac{b(b-a)}{2 \Omega_{0}}\left(\Omega_{1}-\frac{b(a+2 m+4)}{2 m \Omega_{0}(b-a)}\right)\right) R_{C} \cos \theta_{C}=0
\end{aligned}
$$

Solving (89) and (90) results in the following five equilibrium points.

$$
\begin{array}{ll}
R_{C 1}=0 & \theta_{C}=\frac{\pi}{2}, \frac{3 \pi}{2} \\
\left.R_{C 2,3}^{2}=\frac{b(b-a)}{12 \alpha \Omega_{0}}\left(\Omega_{1}+\frac{b(a+2 m+4)}{2 m \Omega_{0}(b-a)}\right)\right) & \theta_{C}=0, \pi \\
\left.R_{C 4,5}^{2}=\frac{b(b-a)}{12 \alpha \Omega_{0}}\left(\Omega_{1}-\frac{b(a+2 m+4)}{2 m \Omega_{0}(b-a)}\right)\right) &
\end{array}
$$

Such that $R_{C 1}$ is corresponded to the trivial equilibria at origin and each of $R_{C 2,3}$ and $R_{C 4,5}$ is corresponded to two nontrivial equilibria located $\pi(\mathrm{rad})$ apart from each other. The local stability of these equilibrium points is determined by the eigenvalues of the Jacobian matrix corresponded to (87) and (88). 
$J=\left[\begin{array}{cc}+12 \alpha C_{1} C_{2} & +6 \alpha\left(C_{1}^{2}+3 C_{2}^{2}\right)-\frac{b(b-a)}{2 \Omega_{0}}\left(\Omega_{1}-\frac{b(a+2 m+4)}{2 m \Omega_{0}(b-a)}\right) \\ -6 \alpha\left(3 C_{1}^{2}+C_{2}^{2}\right)+\frac{b(b-a)}{2 \Omega_{0}}\left(\Omega_{1}+\frac{b(a+2 m+4)}{2 m \Omega_{0}(b-a)}\right) & -12 \alpha C_{1} C_{2}\end{array}\right]$

The $\operatorname{Tr}$ of Eq. (94) is always equal to zero again and the value of the Det is expressed by the following equation.

$$
\begin{aligned}
\text { Det }=108 \alpha^{2}\left(C_{1}^{2}+C_{2}^{2}\right)^{2} & -6 \alpha \frac{b(b-a)}{2 \Omega_{0}}\left(4 \Omega_{1}\left(C_{1}^{2}+C_{2}^{2}\right)-\frac{b(a+2 m+4)}{m \Omega_{0}(b-a)}\left(C_{1}^{2}-C_{2}^{2}\right)\right) \\
& +\frac{b^{2}(b-a)^{2}}{4 \Omega_{0}^{2}}\left(\Omega_{1}^{2}-\frac{b^{2}(a+2 m+4)^{2}}{4 m^{2} \Omega_{0}^{2}(b-a)^{2}}\right)
\end{aligned}
$$

Transforming Eq. (95) into the polar coordinate and substituting Eqs. (91)(93) in it yields the following expression for Det at the equilibrium points.

$$
\begin{aligned}
& \text { Det }=+\frac{b^{2}(b-a)^{2}}{4 \Omega_{0}^{2}}\left(\Omega_{1}^{2}-\frac{b^{2}(a+2 m+4)^{2}}{4 m^{2} \Omega_{0}^{2}(b-a)^{2}}\right) \\
& \text { Det }=+\frac{b^{3}(a+2 m+4)(b-a)}{2 m \Omega_{0}^{3}}\left(\Omega_{1}+\frac{b(a+2 m+4)}{2 m \Omega_{0}(b-a)}\right) \\
& \text { Det }=-\frac{b^{3}(a+2 m+4)(b-a)}{2 m \Omega_{0}^{3}}\left(\Omega_{1}-\frac{b(a+2 m+4)}{2 m \Omega_{0}(b-a)}\right)
\end{aligned}
$$

The Det of the equilibrium points for different values of $\Omega_{1}$ are demonstrated in Fig. 4.
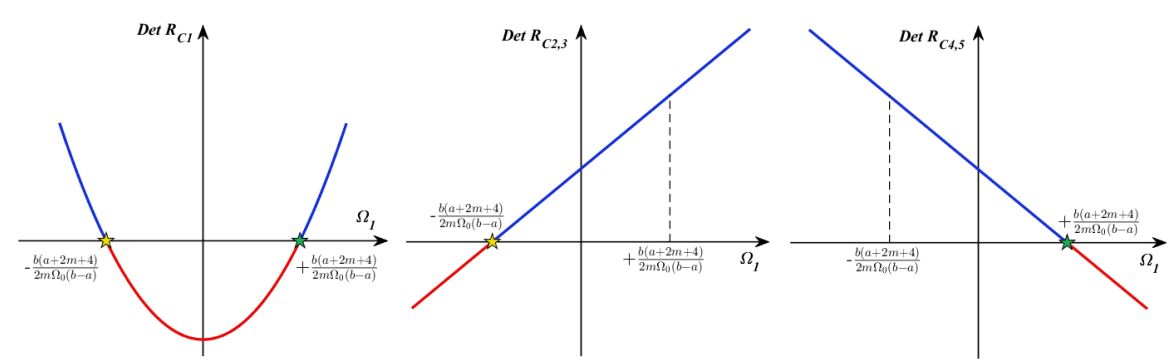

Fig. 4 Determinant at the equilibrium points around the resonance tongue due to the third natural frequency

Besides, Eqs. (92) and (93) provide the square value of the equilibrium points which impose a condition on the value of $\Omega_{1}$. Therefore, for the case of hardening cubic nonlinearity $(\alpha>0)$ the existence of the real equilibria requires that 


$$
\begin{array}{llll}
R_{C 1} & \text { Exist } & \forall & \omega_{1} \\
R_{C 2,3} & \text { Exist } & \forall & \omega_{1}>-\frac{b(a+2 m+4)}{2 m \Omega_{0}(b-a)} \\
R_{C 4,5} & \text { Exist } & \forall & \omega_{1}>+\frac{b(a+2 m+4)}{2 m \Omega_{0}(b-a)}
\end{array}
$$

and for the case of softening cubic nonlinearity $(\alpha<0)$

$$
\begin{array}{llll}
R_{C 1} & \text { Exist } & \forall & \omega_{1} \\
R_{C 2,3} & \text { Exist } & \forall & \omega_{1}<-\frac{b(a+2 m+4)}{2 m \Omega_{0}(b-a)} \\
R_{C 4,5} & \text { Exist } & \forall & \omega_{1}<+\frac{b(a+2 m+4)}{2 m \Omega_{0}(b-a)}
\end{array}
$$

The corresponding bifurcation diagram for hardening and softening cases are plotted in Fig. 5.

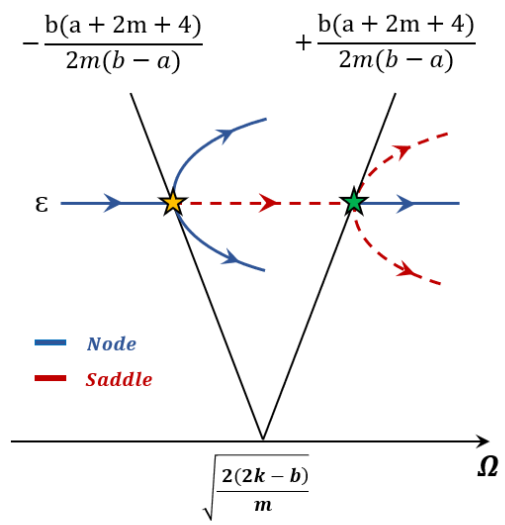

Hardening nonlinearity

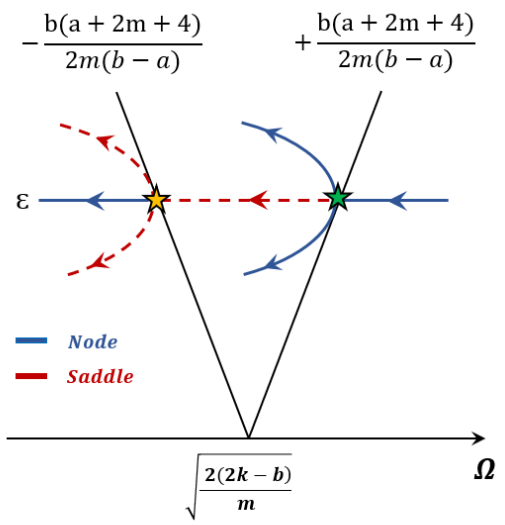

Softening nonlinearity

Fig. 5 Subcritical and supercritical pitchfork bifurcations around the resonance tongue due to the third natural frequenc

This analysis show that, as one crosses the transition curves of the unstable tongue (for the constant value of $\epsilon$ ) the supercritical and subcritical pitchfork bifurcation occurs. Such that for the hardening nonlinearity, origin is the only equilibria at the left-hand side of the unstable tongue and by quasi statically increasing the value of the $\Omega_{1}$, Det of the Jacobian matrix at $R_{C 1}$ and $R_{C 2,3}$ cross the $\Omega_{1}$ axis and results in a supercritical pitchfork bifurcation. Consequently, a subcritical pitchfork bifurcation happens when Det of the Jacobian 
matrix at $R_{C 1}$ and $R_{C 4,5}$ cross the $\Omega_{1}$ axis. Similarly, for the softening nonlinearity, the origin is the only equilibria at the right-hand side of the unstable tongue and by quasi statically decreasing $\Omega_{1}$, Det of $R_{C 1}$ and $R_{C 4,5}$ cross the $\Omega_{1}$ axis and cause a supercritical pitchfork bifurcation. Consequently, a subcritical pitchfork bifurcation happens when Det of Jacobian matrix at $R_{C 1}$ and $R_{C 2,3}$ cross the $\Omega_{1}$ axis.

3.3 Combined Parametric Resonance of the Summation Type Due to the Second and Third Natural Frequencies

For the following special case of the second and third natural frequencies more terms in Eq. (48) become secular.

$$
\begin{aligned}
& \omega_{n 2}-1=-\omega_{n 3} \rightarrow \omega_{n 2}+\omega_{n 3}=1 \\
& \omega_{n 3}-1=-\omega_{n 2} \rightarrow \omega_{n 2}+\omega_{n 3}=1
\end{aligned}
$$

Imposing this condition to Eq. (44) gives the emanating frequency of the unstable tongue related to the combined parametric resonance.

$$
\Omega_{0}^{2}=\frac{(m+k+2)}{m}+2 \sqrt{\frac{k}{m}}
$$

By substituting Eq. (105) in Eq. (48), the following matrix form of the secular terms is obtained.

$$
\begin{aligned}
& {\left[\begin{array}{r}
+2 m \Omega_{0} \Omega_{1} \omega_{n 1}^{2} A_{1}-\frac{3}{4} \alpha X^{2} A_{1}-4 m \Omega_{0} \Omega_{1} \omega_{n 2}^{2} B_{1}+3 \alpha Y^{2} B_{1}-\frac{b}{2} C_{1}-4 m \Omega_{0} \Omega_{1} \omega_{n 3}^{2} C_{1}+3 \alpha Z^{2} C_{1}-\frac{a}{2} B_{1} \\
+2 m \Omega_{0} \Omega_{1} \omega_{n 1}^{2} A_{1}-\frac{3}{4} \alpha X^{2} A_{1}+4 m \Omega_{0} \Omega_{1} \omega_{n 2}^{2} B_{1}-3 \alpha Y^{2} B_{1}+\frac{b}{2} C_{1}+4 m \Omega_{0} \Omega_{1} \omega_{n 3}^{2} C_{1}-3 \alpha Z^{2} C_{1}+\frac{a}{2} B_{1} \\
0 \quad+2 \Omega_{0} \Omega_{1}(a+4) \omega_{n 2}^{2} B_{1}-\frac{b}{2} C_{1}+2 \Omega_{0} \Omega_{1}(b+4) \omega_{n 3}^{2} C_{1}-\frac{a}{2} B_{1}
\end{array}\right]}
\end{aligned}
$$

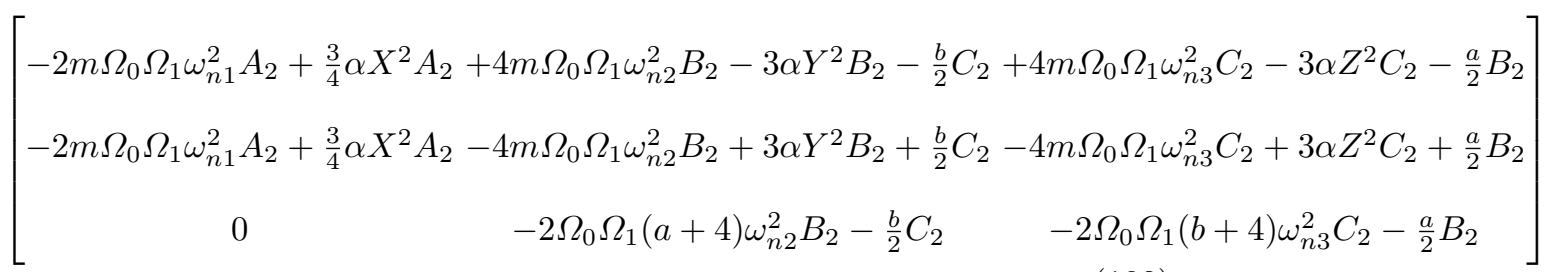

Imposing the zero determinant condition to Eqs, (107) and (108) where $\alpha=0$, results in the following equations. 
$+b(a+2 m+4) \omega_{2}^{2} B_{2}^{2}+8 m \Omega_{0} \Omega_{1}(b-a) \omega_{2}^{2} \omega_{3}^{2} B_{2} C_{2}-a(b+2 m+4) \omega_{3}^{2} C_{2}^{2}=0$

$-b(a+2 m+4) \omega_{2}^{2} B_{1}^{2}+8 m \Omega_{0} \Omega_{1}(b-a) \omega_{2}^{2} \omega_{3}^{2} B_{1} C_{1}+a(b+2 m+4) \omega_{3}^{2} C_{1}^{2}=0$

Each one of Eqs. (109) and (110) form a degenerate conic with standard form of $A x_{1}^{2}+B x_{1} x_{2}+C x_{2}^{2}=0$. Imposing the condition of $A B+2 A C+B C=0$ to these equations provides the two first-order multipliers of Eq. (31) for the transition curves of the unstable tongue. Such that the negative and positive signs are related to sine and cosine multipliers associated to the left and right transition curves, respectively.

$$
\Omega_{1}= \pm \frac{4 k \Omega_{0}}{(a-b)^{2}}
$$

In this case, none of the columns in Eqs. (107) and (108) are in the column space of Eq. (35) for a nontrivial equilibrium point. But one can investigate the change of the stability of the origin by imposing Eq. (57) to the second and third columns, which results in the following four equations.

$$
\left(-4 m \Omega_{0} \Omega_{1} \omega_{n 2}^{2} B_{2}+3 \alpha Y^{2} B_{2}+\frac{b}{2} C_{2}\right)+\left(\frac{a}{4 \Omega_{0}^{2} \omega_{n 2}^{2}}\right)\left(-2 \Omega_{0} \Omega_{1}(a+4) \omega_{n 2}^{2} B_{2}-\frac{b}{2} C_{2}\right)=0
$$

$\left(+4 m \Omega_{0} \Omega_{1} \omega_{n 2}^{2} B_{1}-3 \alpha Y^{2} B_{1}+\frac{b}{2} C_{1}\right)+\left(\frac{a}{4 \Omega_{0}^{2} \omega_{n 2}^{2}}\right)\left(+2 \Omega_{0} \Omega_{1}(a+4) \omega_{n 2}^{2} B_{1}-\frac{b}{2} C_{1}\right)=0$

$$
\left(-4 m \Omega_{0} \Omega_{1} \omega_{n 3}^{2} C_{2}+3 \alpha Z^{2} C_{2}+\frac{a}{2} B_{2}\right)+\left(\frac{b}{4 \Omega_{0}^{2} \omega_{n 3}^{2}}\right)\left(-2 \Omega_{0} \Omega_{1}(b+4) \omega_{n 3}^{2} C_{2}-\frac{a}{2} B_{2}\right)=0
$$

$\left(+4 m \Omega_{0} \Omega_{1} \omega_{n 3}^{2} C_{1}-3 \alpha Z^{2} C_{1}+\frac{a}{2} B_{1}\right)+\left(\frac{b}{4 \Omega_{0}^{2} \omega_{n 3}^{2}}\right)\left(+2 \Omega_{0} \Omega_{1}(b+4) \omega_{n 3}^{2} C_{1}-\frac{a}{2} B_{1}\right)=0$

Equations (112)-(115) can be simplified as follow by using the relationship between parameters $a, b$ and the second and third natural frequencies. 


$$
\begin{aligned}
& \left(+6 \alpha\left(B_{1}^{2}+B_{2}^{2}\right)+12 \alpha\left(C_{1}^{2}+C_{2}^{2}\right)-\frac{a(a-b) \Omega_{1}}{2 \Omega_{0}}\right) B_{2}+k C_{2}=0 \\
& \left(-6 \alpha\left(B_{1}^{2}+B_{2}^{2}\right)-12 \alpha\left(C_{1}^{2}+C_{2}^{2}\right)+\frac{a(a-b) \Omega_{1}}{2 \Omega_{0}}\right) B_{1}+k C_{1}=0 \\
& \left(+12 \alpha\left(B_{1}^{2}+B_{2}^{2}\right)+6 \alpha\left(C_{1}^{2}+C_{2}^{2}\right)+\frac{b(a-b) \Omega_{1}}{2 \Omega_{0}}\right) C_{2}+k B_{2}=0 \\
& \left(-12 \alpha\left(B_{1}^{2}+B_{2}^{2}\right)-6 \alpha\left(C_{1}^{2}+C_{2}^{2}\right)-\frac{b(a-b) \Omega_{1}}{2 \Omega_{0}}\right) C_{1}+k B_{1}=0
\end{aligned}
$$

The local stability of the origin is determined by the eigenvalues of the Jacobian matrix corresponded to Eqs. (116) - (119).

$$
J=\left[\begin{array}{cccc}
0 & -\frac{a(a-b) \Omega_{1}}{2 \Omega_{0}} & 0 & k \\
+\frac{a(a-b) \Omega_{1}}{2 \Omega_{0}} & 0 & k & 0 \\
0 & k & 0 & +\frac{b(a-b) \Omega_{1}}{2 \Omega_{0}} \\
k & 0 & -\frac{b(a-b) \Omega_{1}}{2 \Omega_{0}} & 0
\end{array}\right]
$$

One can find the four eigenvalues of Eq. (120) with the following equation.

$$
\lambda^{2}= \pm \frac{a-b}{2 \Omega_{0}} \sqrt{\left(\frac{4 k^{2} \Omega_{0}^{2}}{(a-b)^{2}}-\frac{\left(a^{2}-b^{2}\right)}{2} \Omega_{1}^{2}\right) \pm \sqrt{\left(\frac{4 k^{2} \Omega_{0}^{2}}{(a-b)^{2}}-\frac{\left(a^{2}-b^{2}\right)}{2} \Omega_{1}^{2}\right)^{2}-\left(\frac{4 k^{2} \Omega_{0}^{2}}{(a-b)^{2}}+a b \Omega_{1}^{2}\right)^{2}}}
$$

As long as the following condition is satisfied, all the four eigenvalues expressed by Eq. (121) are pure imaginary with no real part which makes the origin a stable node.

$$
\left(\frac{4 k^{2} \Omega_{0}^{2}}{(a-b)^{2}}-\frac{\left(a^{2}-b^{2}\right)}{2} \Omega_{1}^{2}\right)^{2}-\left(\frac{4 k^{2} \Omega_{0}^{2}}{(a-b)^{2}}+a b \Omega_{1}^{2}\right)^{2}>0
$$

But, in the following region, Eq. (122) is violated and the eigenvalues have both real and imaginary parts. Such that two of the eigenvalues have negative real part and two have positive real parts. In such a condition origin is an unstable spiral [12].

$$
-\frac{4 k \Omega_{0}}{(a-b)^{2}}<\Omega_{1}<+\frac{4 k \Omega_{0}}{(a-b)^{2}}
$$

The eigenvalues of Eq. (120) at origin for different values of $\Omega_{1}$ are demonstrated in Fig. 6. 

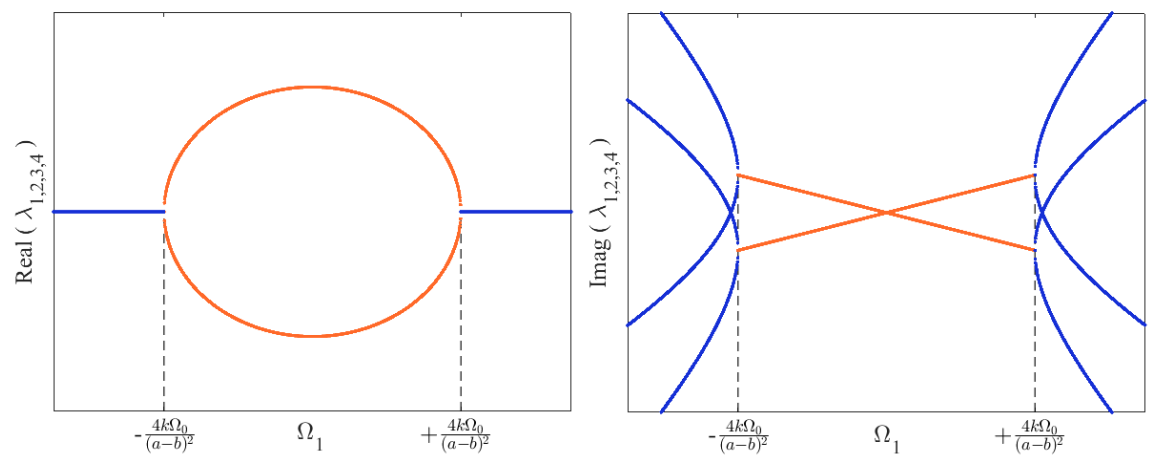

Fig. 6 Real and imaginary parts of the eigenvalues of Eq. (120)

The corresponding bifurcation diagram is plotted in Fig. 7 which is valid for both hardening and softening cases.

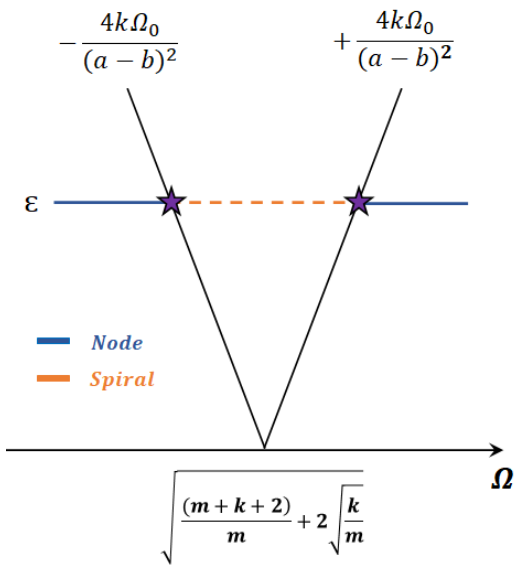

Fig. 7 Subcritical and supercritical hopf bifurcations around the resonance tongue due to summation of the second and third natural frequencies

This analysis show that, as one crosses the transition curves of the unstable tongue (for the constant value of $\epsilon$ ) the supercritical and subcritical hopf bifurcation occurs. Such that for the hardening nonlinearity, by crossing the left-hand side transition curve of the unstable tongue a limit cycle is created by occurrence of a supercritical hopf bifurcation. By quasi statically increasing the value of the $\Omega_{1}$ the radius of this limit cycle increases until by crossing the right hand-side transition curve of the unstable tongue the limit cycle is destroyed by occurrence of a subcritical hopf bifurcation. Similarly, the same sequence of events happens for the softening case, only by quasi statically decreasing the value of the $\Omega_{1}$. 


\subsection{Overall Bifurcation}

Figure 8 shows how the eigenvalues of the Jacobian matrix at origin change for the hardening case while the value of $\Omega$ is being increased. Figure 8-a show that starting from the unstable tongue corresponded to $w_{n 2}$ the origin is a saddle as the eigenvalues are real with both positive and negative signs. Figure 8-a show that by increasing the value of $\Omega$ the real eigenvalues approach origin. By crossing the right-hand side transition curve, the real eigenvalues cross the origin and become pure complex, in such a case the origin is a stable node. Figure 8-a show that by further increase in the value of $\Omega$ the eigenvalues get more distant from the origin. At this point by increasing $\Omega$ and crossing the right transition curve of the unstable tongue corresponded to $w_{n 2}+w_{n 3}$ the eigenvalues leave the imaginary axis. In such a condition the eigenvalues have both real and imaginary parts and due to the eigenvalues with positive real part the origin is an unstable spiral. Figure 8-a show that by more increase in the value of $\Omega$, the imaginary part of the eigenvalues get smaller while the real parts get larger. By reaching the middle of the $w_{n 2}+w_{n 3}$ unstable tongue where $\Omega_{1}=0$ the imaginary parts of the eigenvalues get very close to zero, but as it is shown in Fig. 8-b by passing the middle of the $w_{n 2}+w_{n 3}$ unstable tongue, the imaginary part of the eigenvalues start to increase again while the real part get smaller. This continues until by passing the right transition curve of $w_{n 2}+w_{n 3}$ unstable tongue the eigenvalues become pure complex again which makes the origin a stable node again. Finally, Fig 8-b shows that by more increase in the value of $\Omega$ and crossing the left transition curve of the unstable tongue corresponded to $w_{n 3}$ the origin become a saddle again due to the real eigenvalues with both positive and negative signs.

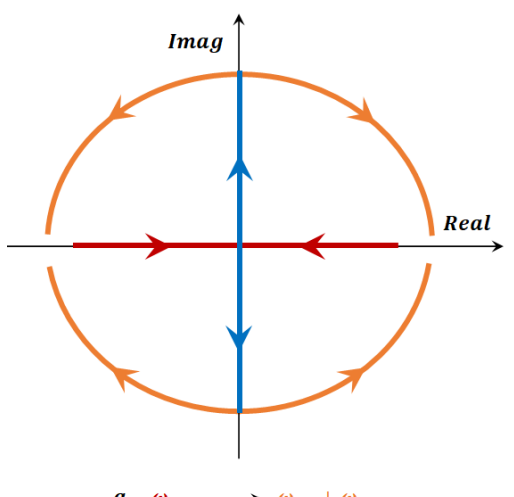

a. $\omega_{n 2} \longrightarrow \omega_{n 2}+\omega_{n 3}$

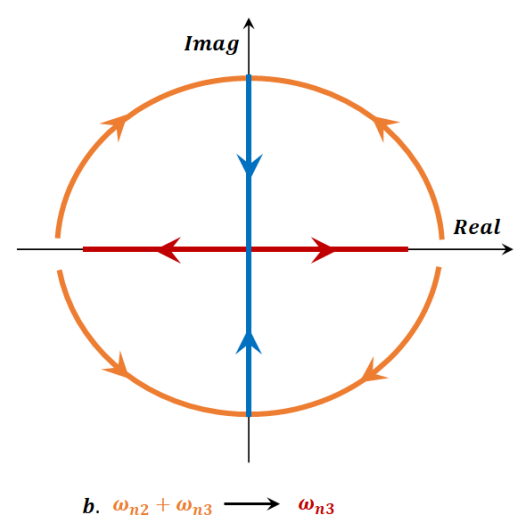

b. $\omega_{n 2}+\omega_{n 3} \longrightarrow \omega_{n 3}$

Fig. 8 Eigenvalues of the Jacobian matrix at origin for the hardening nonlinearity

Figs. 9 and 10 demonstrate the global bifurcation diagram of the hardening and softening nonlinear cases, respectively. 

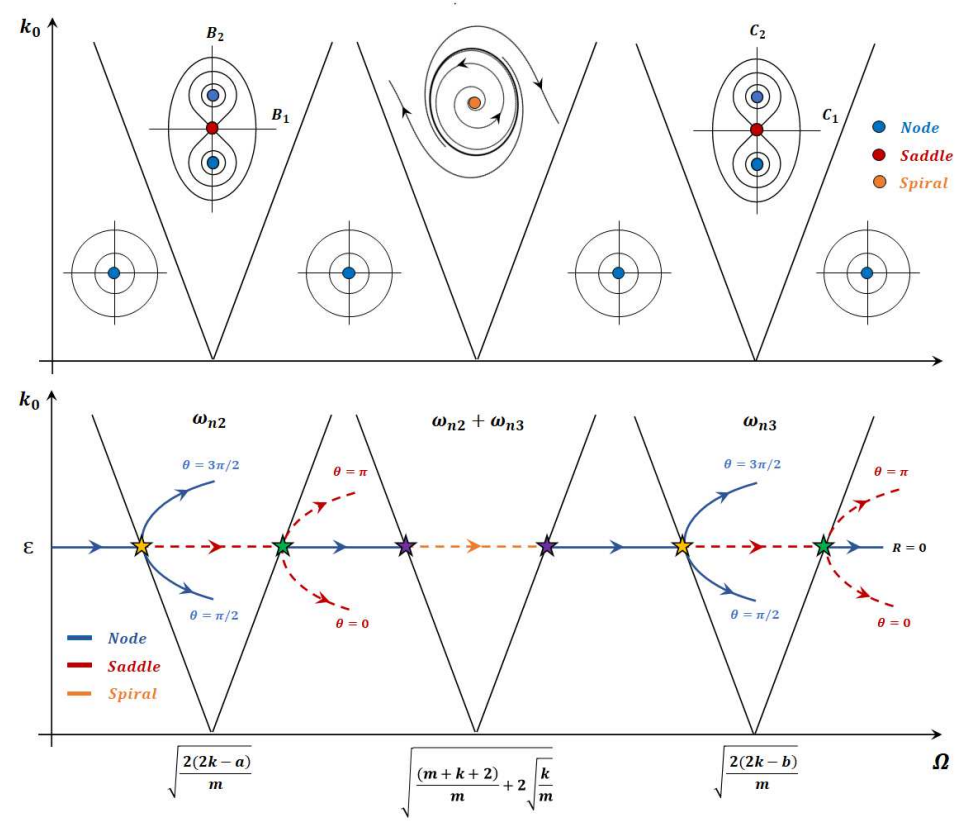

Fig. 9 Global Bifurcation Diagram for the Hardening Nonlinearity
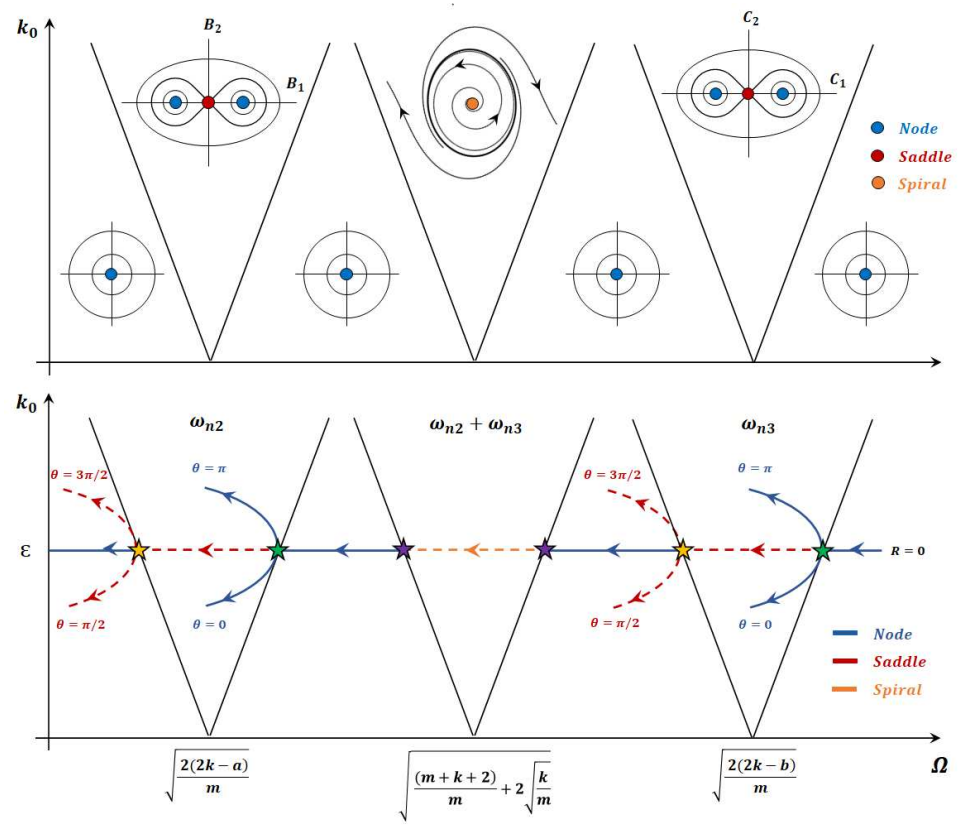

Fig. 10 Global Bifurcation Diagram for the Softening Nonlinearity 


\section{Numerical Simulations}

In this section, the foregoing analytical analysis is compared with the results obtained from numerical integration which is completed in MATLAB by using Runge-Kutta of the fourth-fifth order.

\subsection{Poincare Map}

Figures 11-19 exhibit the Poincare map of Eq. (21) for the softening nonlinearity, all for the surface of section at $t=\omega_{p} / 2 \pi$. Figures 11-13 belongs to the system's parameters inside the unstable tongue corresponded to $\omega_{n 2}$ showing that origin is a saddle and there are two stable nodes around it, $\pi$ ( rad) apart from each other. these plots show that by increasing the value of $\Omega$, the two stable node get closer to the origin which is consistent with the analytical results demonstrated by Fig. 3. Figures 14-16 belongs to the system's parameters inside the unstable tongue corresponded to $\omega_{n 2}+\omega_{n 3}$ showing that origin is an unstable spiral surrounded by a limit cycle. these plots show that by increasing the value of $\Omega$, the size of the limit cycle get smaller. Figures 17-19 belongs to the system's parameters inside the unstable tongue corresponded to $\omega_{n 3}$ showing that origin is a saddle and there are two stable nodes around it, $\pi(\mathrm{rad})$ apart from each other. These plots show that by increasing the value of $\Omega$, the two stable node get closer to the origin which is consistent with the analytical results demonstrated by Fig. 5

\subsection{Time Response}

Figures 20 and 21 demonstrate the effect of softening nonlinear terms on the dynamic response of Eq. (21) inside the unstable tongue corresponded to $\omega_{n 2}$. Comparing these two plots show that, even inside the unstable tongues, by applying enough load on the system and choosing the right parameters for the suspension, the system response can remain bounded and remain outside of the dead zone area. In such a condition, the separation of the teeth in mesh and consequently occurrence of the free play mode and impact phases can be avoided. 

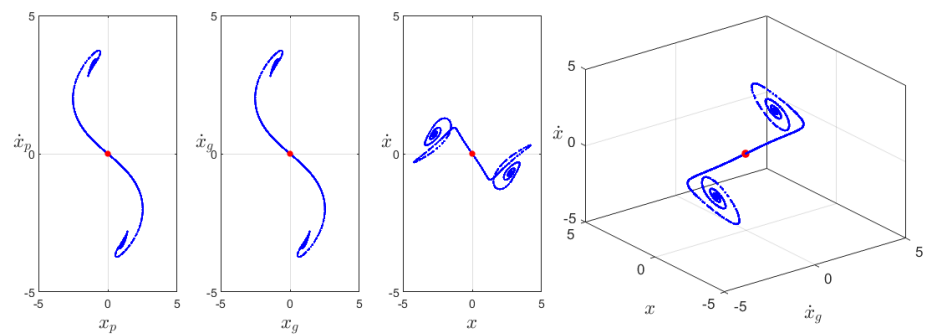

Fig. 11 Poincare map softening, $m=1, \zeta=0.0520, k=5, \alpha=-0.2, k_{0}=0.5, \omega=1.50$
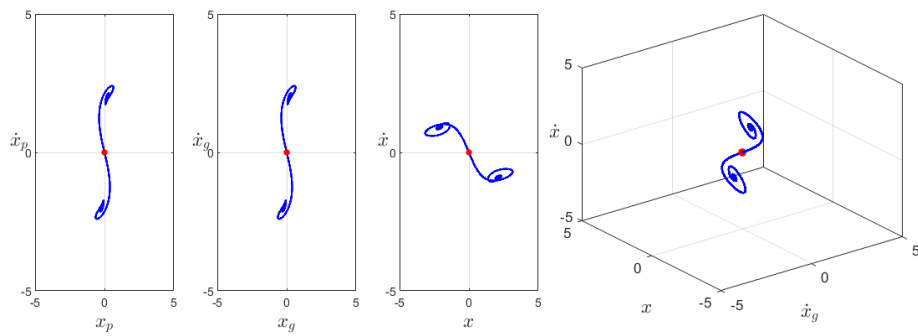

Fig. 12 Poincare map, softening, $m=1, \zeta=0.0300, k=5, \alpha=-0.2, k_{0}=0.5, \omega=1.64$
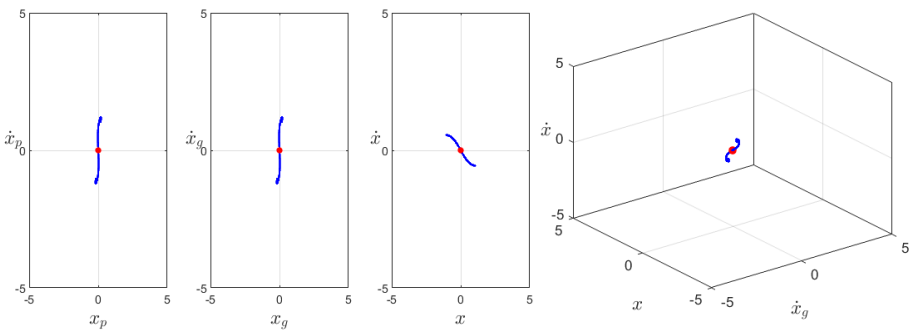

Fig. 13 Poincare map, softening, $m=1, \zeta=0.0200, k=5, \alpha=-0.2, k_{0}=0.5, \omega=1.68$ 
Title Suppressed Due to Excessive Length

29
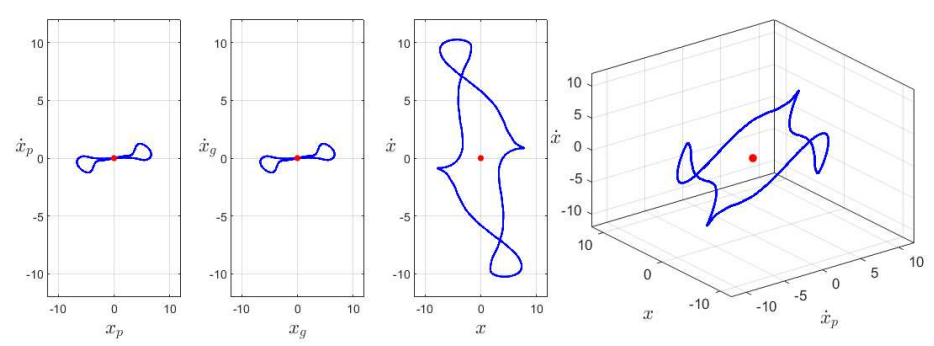

Fig. 14 Poincare map, softening, $m=1, \zeta=0.0100, k=5, \alpha=-0.2, k_{0}=0.5, \omega=3.50$
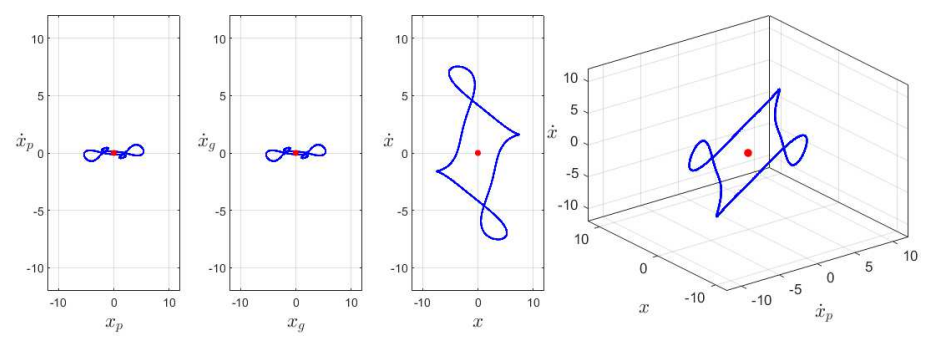

Fig. 15 Poincare map, softening, $m=1, \zeta=0.0100, k=5, \alpha=-0.2, k_{0}=0.5, \omega=3.60$
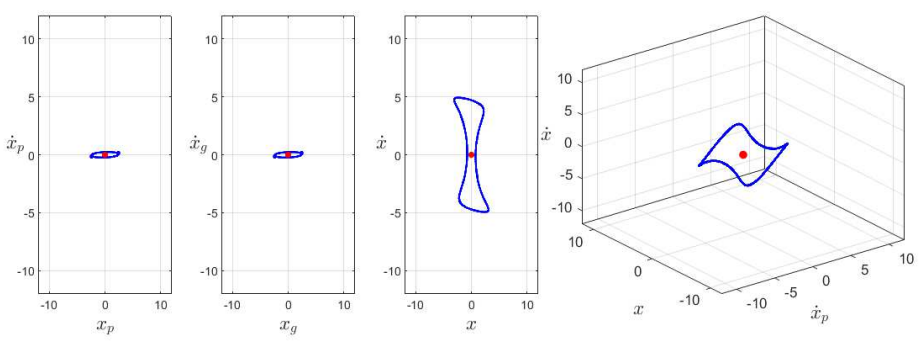

Fig. 16 Poincare map, softening, $m=1, \zeta=0.0200, k=5, \alpha=-0.2, k_{0}=0.5, \omega=3.70$ 

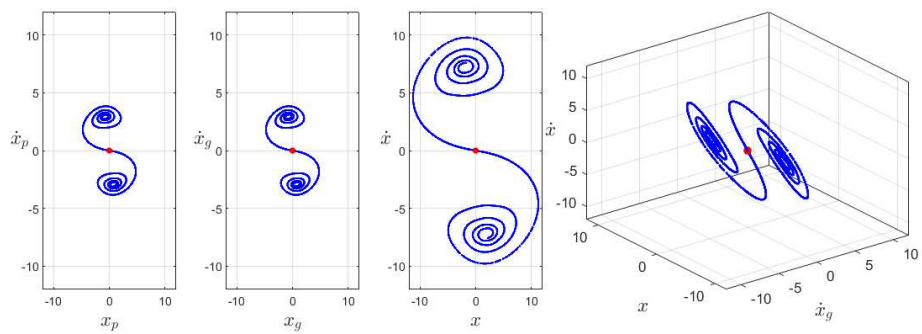

Fig. 17 Poincare map, softening, $m=1, \zeta=0.0075, k=5, \alpha=-0.2, k_{0}=0.5, \omega=5.20$
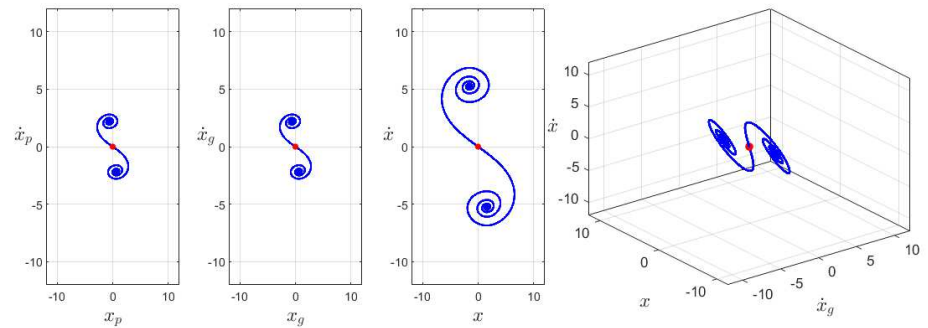

Fig. 18 Poincare map, softening, $m=1, \zeta=0.0075, k=5, \alpha=-0.2, k_{0}=0.5, \omega=5.40$
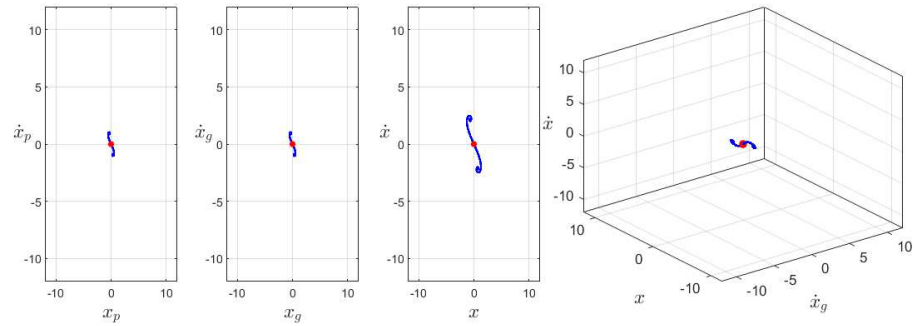

Fig. 19 Poincare map, softening, $m=1, \zeta=0.0075, k=5, \alpha=-0.2, k_{0}=0.5, \omega=5.60$ 

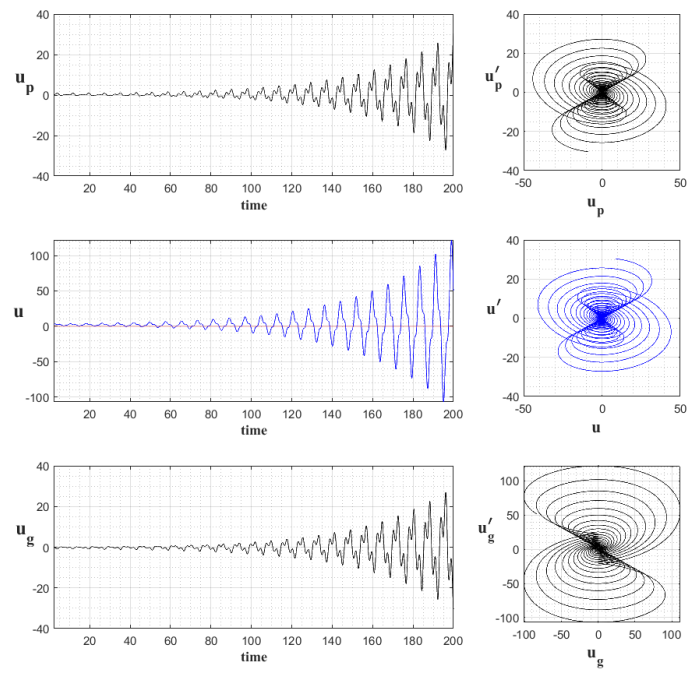

Fig. 20 Time Response, $m=1, \zeta=0.07, k=5, \alpha=0, k_{0}=0.5, F=1, \omega=1.60$
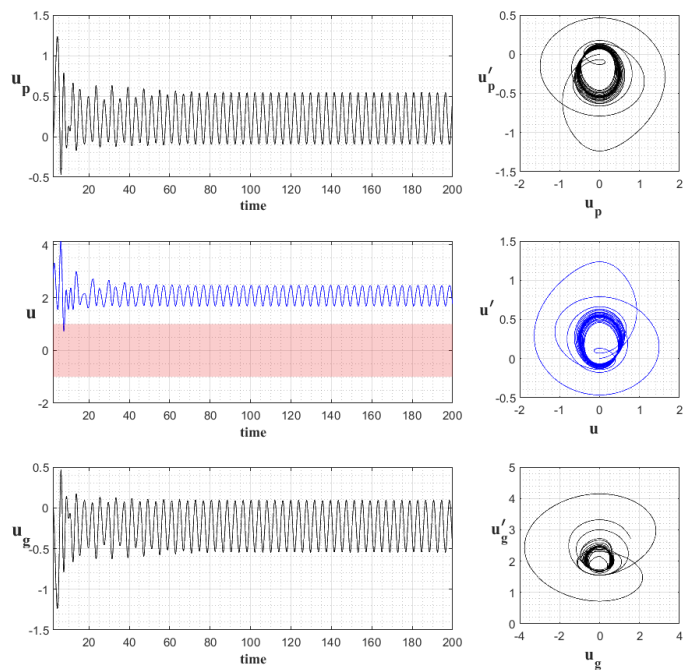

Fig. 21 Time Response, $m=1, \zeta=0.07, k=5, \alpha=-2, k_{0}=0.5, F=1, \omega=1.60$ 


\section{Conclusion}

In this work, Poincare-Lindstedt method is used to study the effect of nonlinear suspension on the dynamic behavior of the geared systems where the transmitting force is large enough that the gears in mesh remain in permanent contact regime. In such a condition, the governing equations reduce to a system of nonlinear parametrically excited coupled differential equations. Such that in the absence of the nonlinear terms the unbounded response of the system happens only within the primary and combined parametric resonance tongues. Where the large amplitude oscillation results in the separation of the gears in mesh and consequently the free play mode and impact phases should be considered in the dynamic response of the system. The analytical and numerical results in this paper show that for the system with cubic nonlinear terms, and for the right range of the parameters the system's response within the unstable tongues can remain bounded. The results show that the bounded response of the system within the unstable tongues is due to the occurrence of the pitchfork bifurcation within the primary unstable tongues and hopf bifurcation within the combined unstable tongue. It is shown that the occurrence of the supercritical and subcritical pitchfork bifurcations follows the same pattern for both hardening and softening nonlinearity, only with a $\pi / 2(\mathrm{rad})$ difference on the location of the newborn equilibria. Poincare map is presented only for the softening case, as the experimental data shows mostly softening nonlinear behaviors of the geared system. The provided Poincare map confirms creation of the new equilibria within the primary unstable tongues and creation of the limit cycle within the combined unstable tongue which was predicted by Poincare-Lindstedt method. It is shown that by change of control parameter the distance between the origin and nodes and also the radius of the limit cycle varies. At the end, the time response of the system for the parameter related to one of the unstable tongues, are provided. These plots show that the small amplitude oscillation is possible even inside the unstable tongues by choosing the right parameter for the suspension which prevent the separation of the gears in mesh. 


\section{Declarations}

Funding: this study is not funded by any organization or company.

Conflicts of interest/Competing interests: the author declare that he has no known competing financial interests or personal relationships that could have appeared to influence the work reported in this paper.

Availability of data and material: Not applicable.

Code availability: Not applicable.

Authors' contributions (optional)

Ethics approval: Not applicable.

Consent to participate: the Author warrants that the Work has not been published before in any form except as a preprint, that the Work is not being concurrently submitted to and is not under consideration by another publisher, that the persons listed above are listed in the proper order and that no author entitled to credit has been omitted.

Consent for publication: The Author hereby transfers to the Publisher the copyright of the Work. As a result, the Publisher shall have the exclusive and unlimited right to publish the Work throughout the World. 


\section{References}

1. Cao, Z., Chen, Z., Jiang, H.: Nonlinear dynamics of a spur gear pair with force-dependent mesh stiffness. Nonlinear Dynamics 99(2), 1227-1241 (2019). DOI 10.1007/s11071-01905348-0. URL https://doi.org/10.1007/s11071-019-05348-0

2. Chang-Jian, C.W.: Nonlinear dynamic analysis for bevel-gear system under nonlinear suspension-bifurcation and chaos. Applied Mathematical Modelling 35(7), 3225-3237 (2011). DOI 10.1016/j.apm.2011.01.027. URL https://doi.org/10.1016/j.apm.2011.01.027

3. Chen, Q., Zhou, J., Khushnood, A., Wu, Y., Zhang, Y.: Modelling and nonlinear dynamic behavior of a geared rotor-bearing system using tooth surface microscopic features based on fractal theory. AIP Advances 9(1), 015201 (2019). DOI 10.1063/1.5055907. URL https://doi.org/10.1063/1.5055907

4. Dadon, I., Koren, N., Klein, R., Bortman, J.: A realistic dynamic model for gear fault diagnosis. Engineering Failure Analysis 84, 77-100 (2018). DOI 10.1016/j.engfailanal.2017.10.012. URL https://doi.org/10.1016/j.engfailanal.2017.10.012

5. Farshidianfar, A., Saghafi, A.: Global bifurcation and chaos analysis in nonlinear vibration of spur gear systems. Nonlinear Dynamics 75(4), 783-806 (2013). DOI 10.1007/s11071-013-1104-4. URL https://doi.org/10.1007/s11071-013-1104-4

6. Li, Z., Peng, Z.: Nonlinear dynamic response of a multi-degree of freedom gear system dynamic model coupled with tooth surface characters: a case study on coal cutters. Nonlinear Dynamics 84(1), 271-286 (2015). DOI 10.1007/s11071-015-2475-5. URL https://doi.org/10.1007/s11071-015-2475-5

7. Margielewicz, J., Gaska, D., Litak, G.: Modelling of the gear backlash. Nonlinear Dynamics 97(1), 355-368 (2019). DOI 10.1007/s11071-019-04973-z. URL https://doi.org/10.1007/s11071-019-04973-z

8. Mason, J.F., Piiroinen, P.T., Wilson, R.E., Homer, M.E.: Basins of attraction in nonsmooth models of gear rattle. International Journal of Bifurcation and Chaos 19(01), 203-224 (2009). DOI 10.1142/s021812740902283x. URL https://doi.org/10.1142/s021812740902283x

9. Morrison, T.M., Rand, R.H.: 2:1 resonance in the delayed nonlinear mathieu equation. Nonlinear Dynamics 50(1-2), 341-352 (2007). DOI 10.1007/s11071-006-9162-5. URL https://doi.org/10.1007/s11071-006-9162-5

10. Nayfeh, A.H., Mook, D.T.: Nonlinear oscillations. Wiley (1995). DOI 10.1002/9783527617586. URL https://doi.org/10.1002/9783527617586

11. Saghafi, A., Farshidianfar, A.: An analytical study of controlling chaotic dynamics in a spur gear system. Mechanism and Machine Theory 96, 179-191 (2016). DOI 10.1016/j.mechmachtheory.2015.10.002. URL https://doi.org/10.1016/j.mechmachtheory.2015.10.002

12. Strogatz, S.H.: Nonlinear Dynamics and Chaos. CRC Press (2018). DOI 10.1201/9780429492563. URL https://doi.org/10.1201/9780429492563

13. Xia, Y., Wan, Y., Chen, T.: Investigation on bifurcation and chaos control for a spur pair gear system with and without nonlinear suspension. In: 2018 37th Chinese Control Conference (CCC). IEEE (2018). DOI 10.23919/chicc.2018.8484012. URL https://doi.org/10.23919/chicc.2018.8484012

14. Xiong, Y., Huang, K., Xu, F., Yi, Y., Sang, M., Zhai, H.: Research on the influence of backlash on mesh stiffness and the nonlinear dynamics of spur gears. Applied Sciences 9(5), 1029 (2019). DOI 10.3390/app9051029. URL https://doi.org/10.3390/app9051029 
Figures

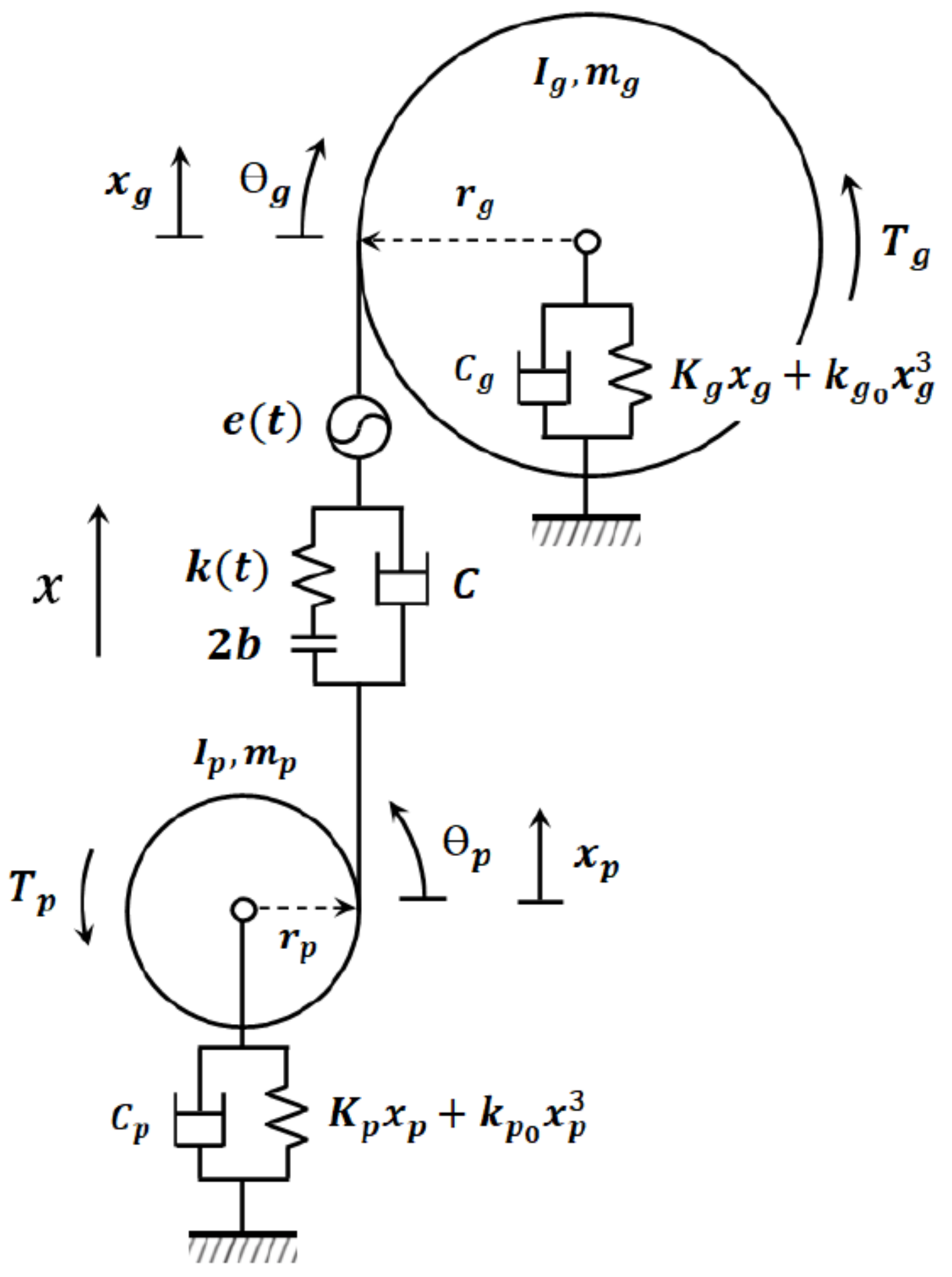

Figure 1

Schematic of pinion and gear in mesh with nonlinear suspension 


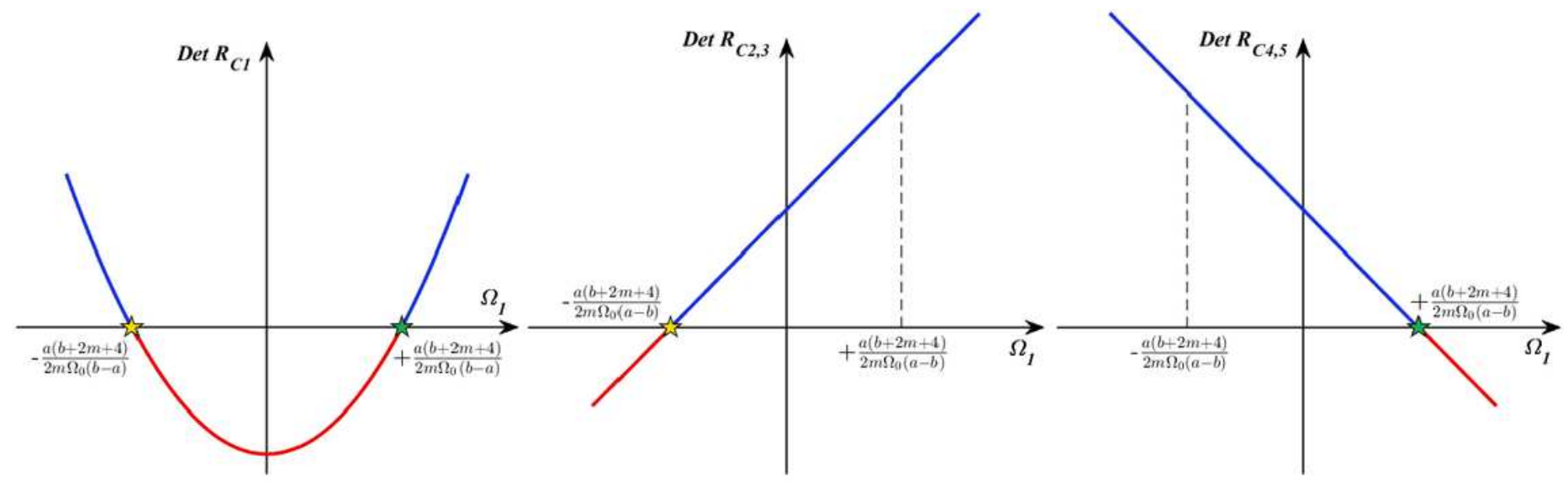

Figure 2

Determinant at the equilibrium points around the resonance tongue due to the second natural frequency

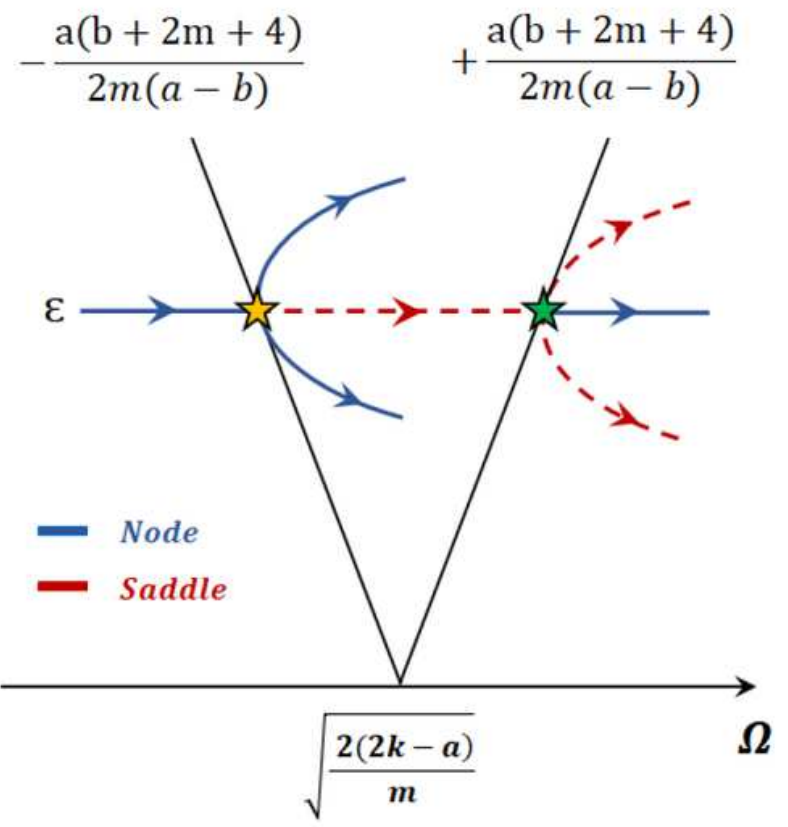

Hardening nonlinearity
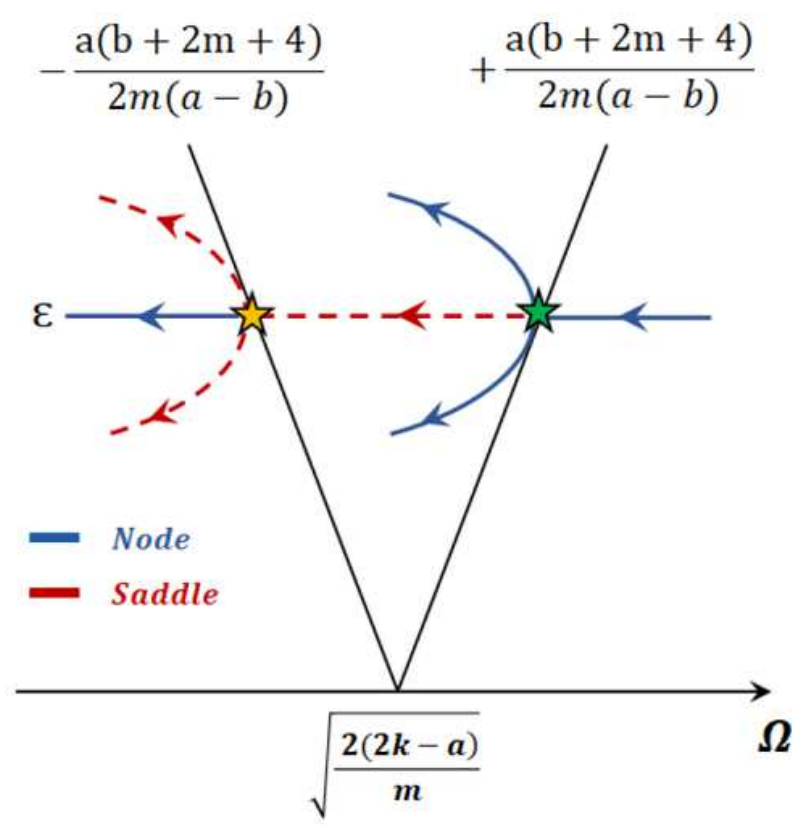

Softening nonlinearity

\section{Figure 3}

Subcritical and supercritical pitchfork bifurcations around the resonance tongue due to the second natural frequenc 

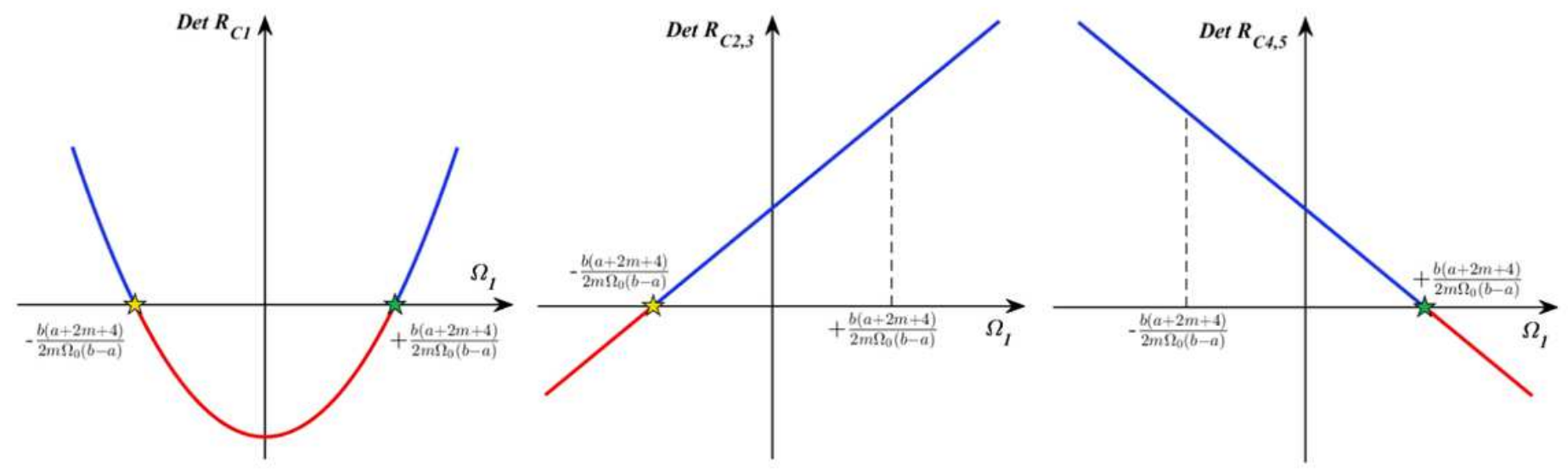

Figure 4

Determinant at the equilibrium points around the resonance tongue due to the third natural frequency

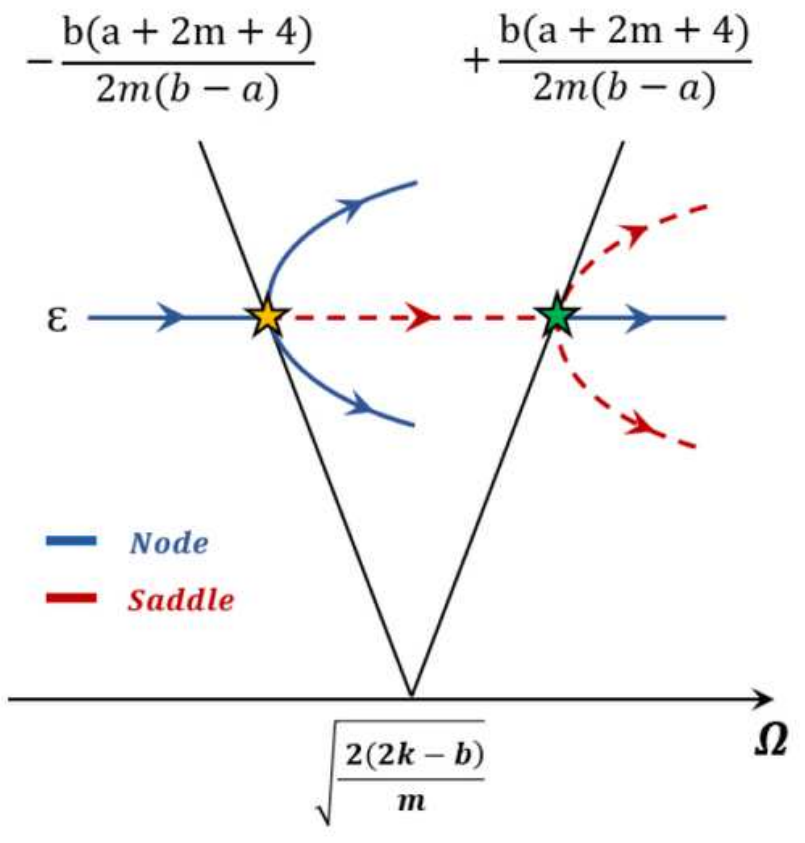

Hardening nonlinearity$$
-\frac{\mathrm{b}(\mathrm{a}+2 \mathrm{~m}+4)}{2 m(b-a)} \quad+\frac{\mathrm{b}(\mathrm{a}+2 \mathrm{~m}+4)}{2 m(b-a)}
$$

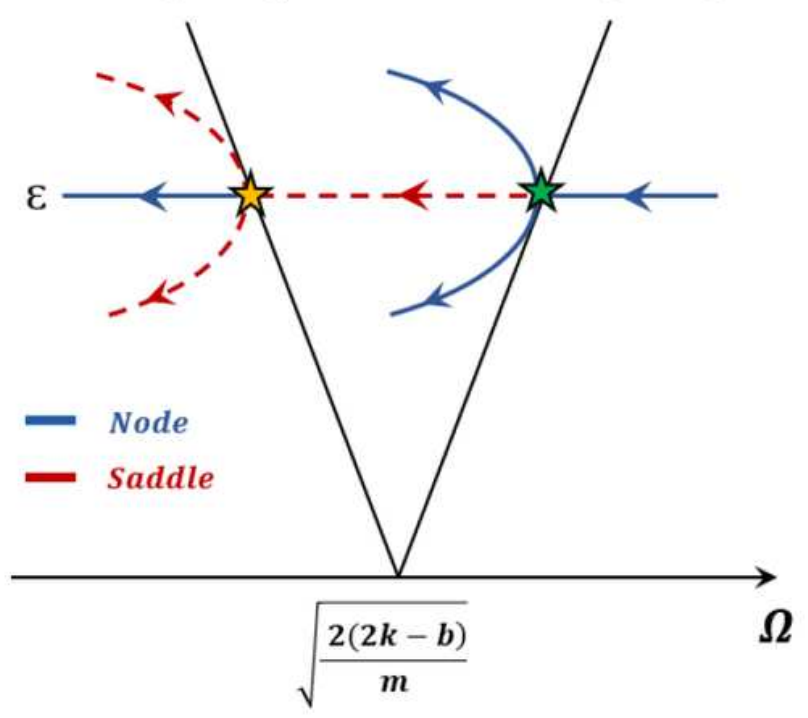

Softening nonlinearity

Figure 5

Subcritical and supercritical pitchfork bifurcations around the resonance tongue due to the third natural frequenc 

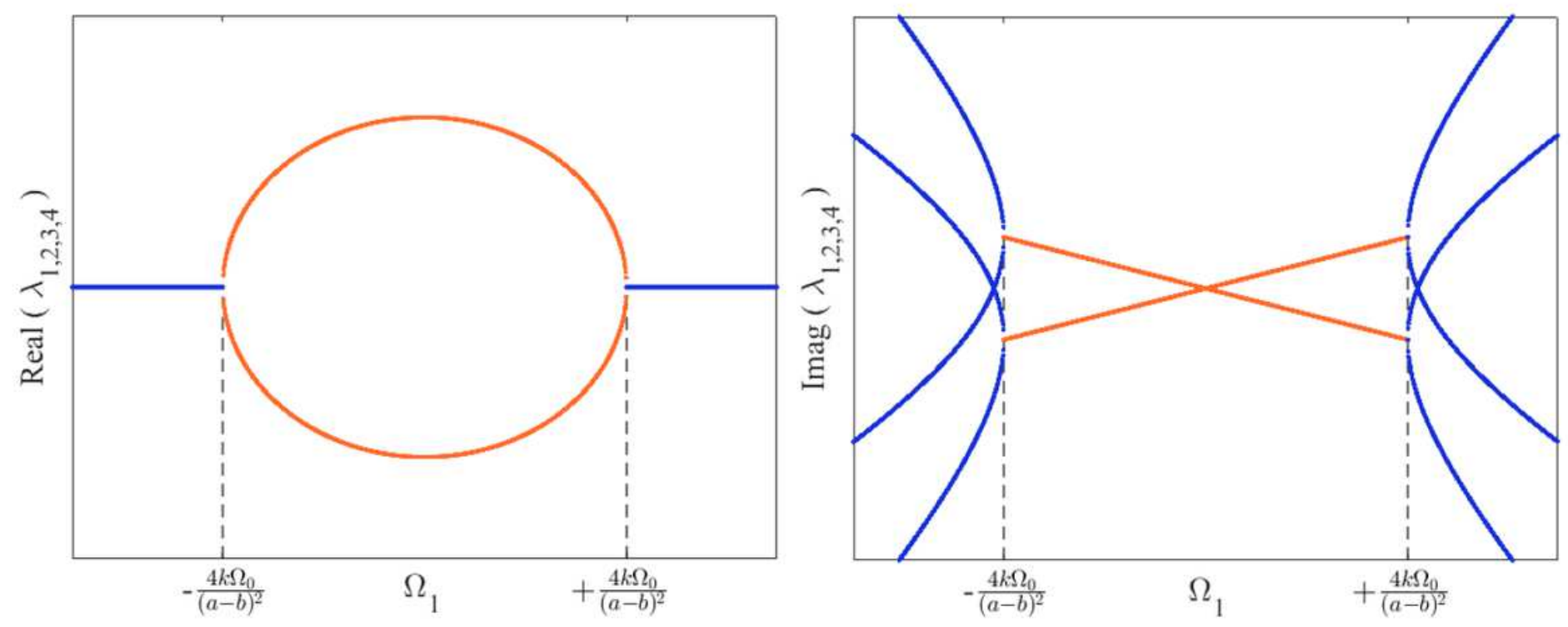

Figure 6

Real and imaginary parts of the eigenvalues of Eq. (120) 


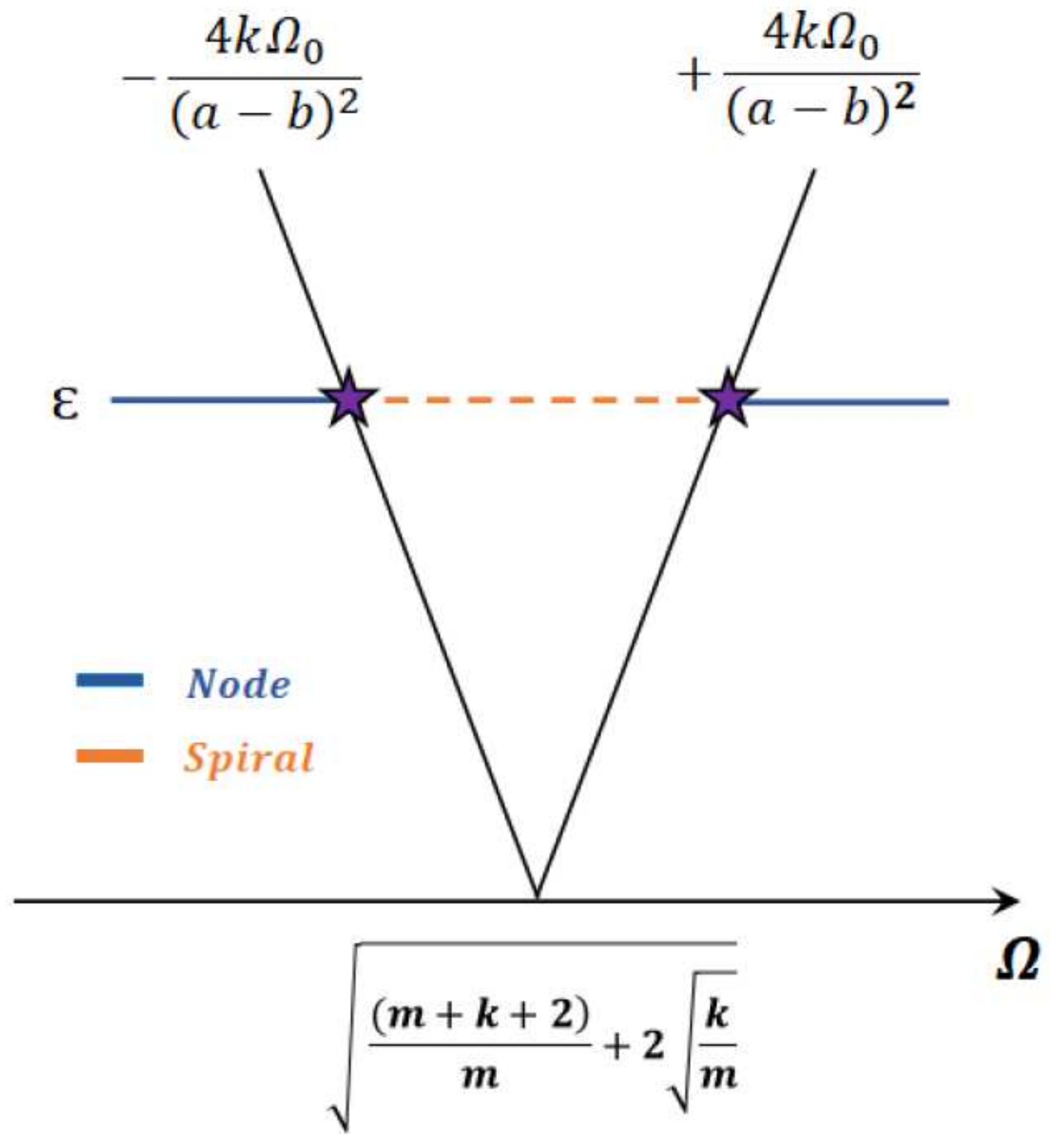

Figure 7

Subcritical and supercritical hopf bifurcations around the resonance tongue due to summation of the second and third natural frequencies 


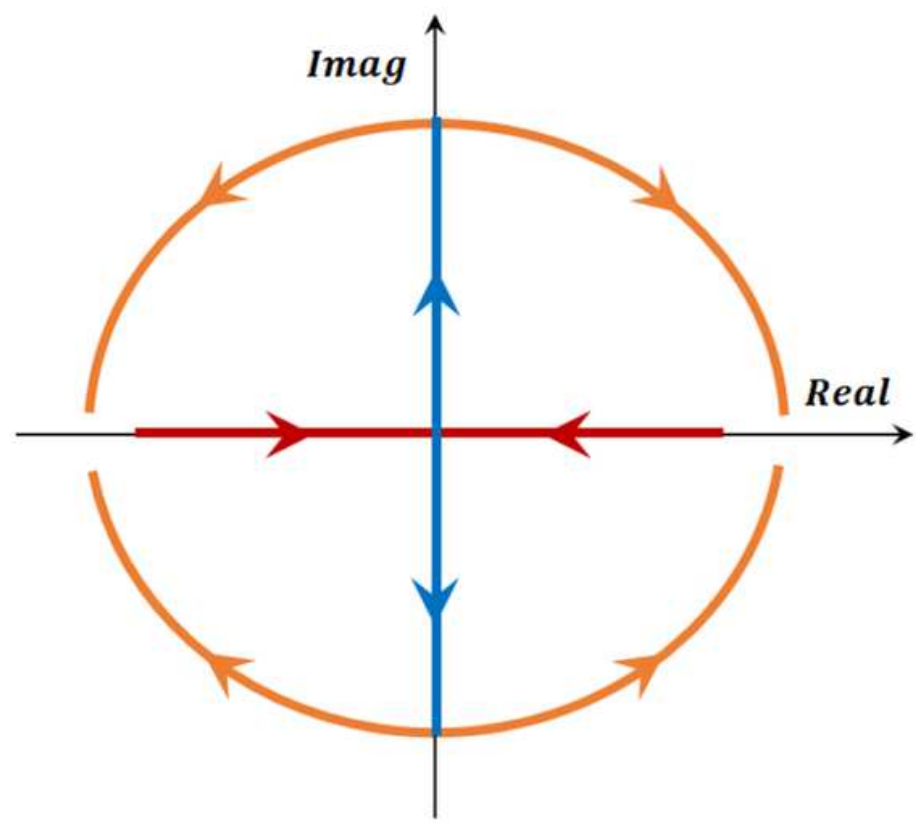

a. $\omega_{n 2} \longrightarrow \omega_{n 2}+\omega_{n 3}$

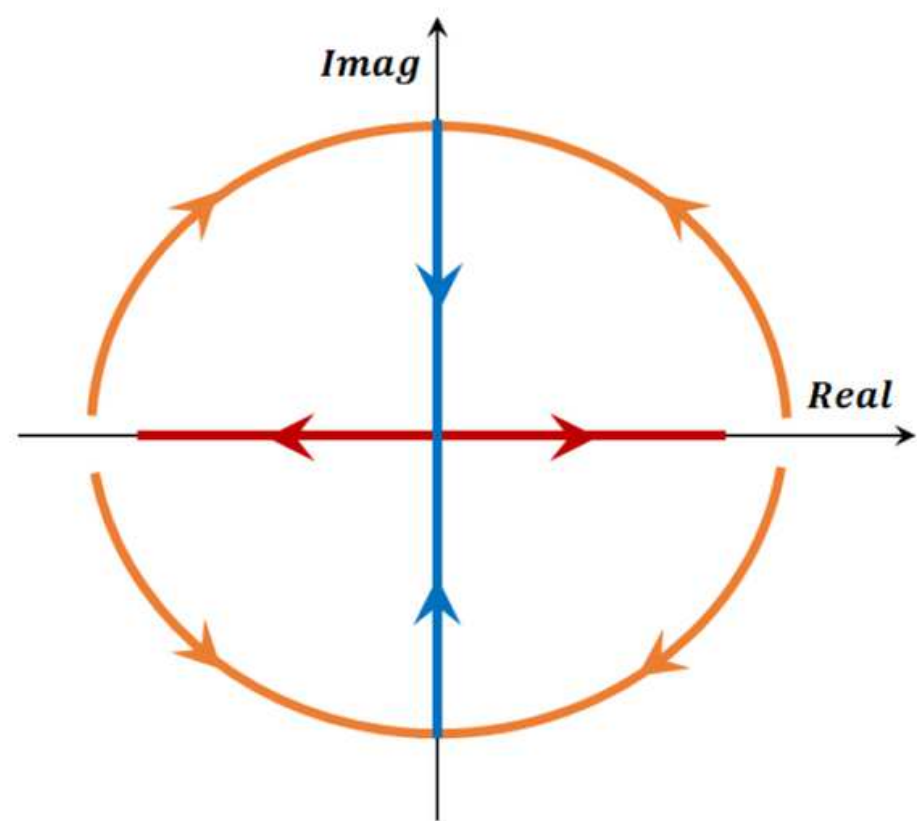

b. $\omega_{n 2}+\omega_{n 3} \longrightarrow \omega_{n 3}$

Figure 8

Eigenvalues of the Jacobian matrix at origin for the hardening nonlinearity 

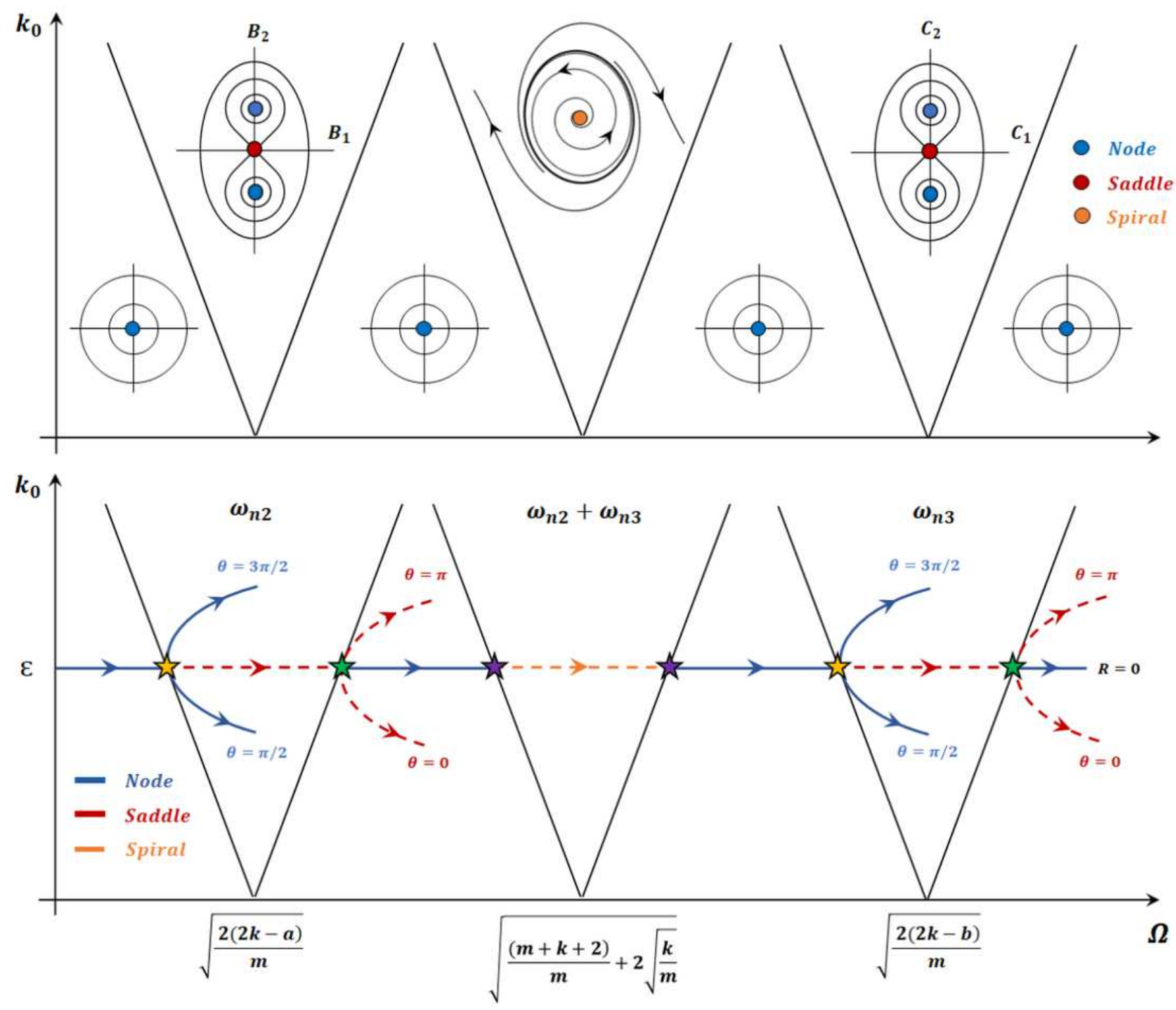

Figure 9

Global Bifurcation Diagram for the Hardening Nonlinearity 

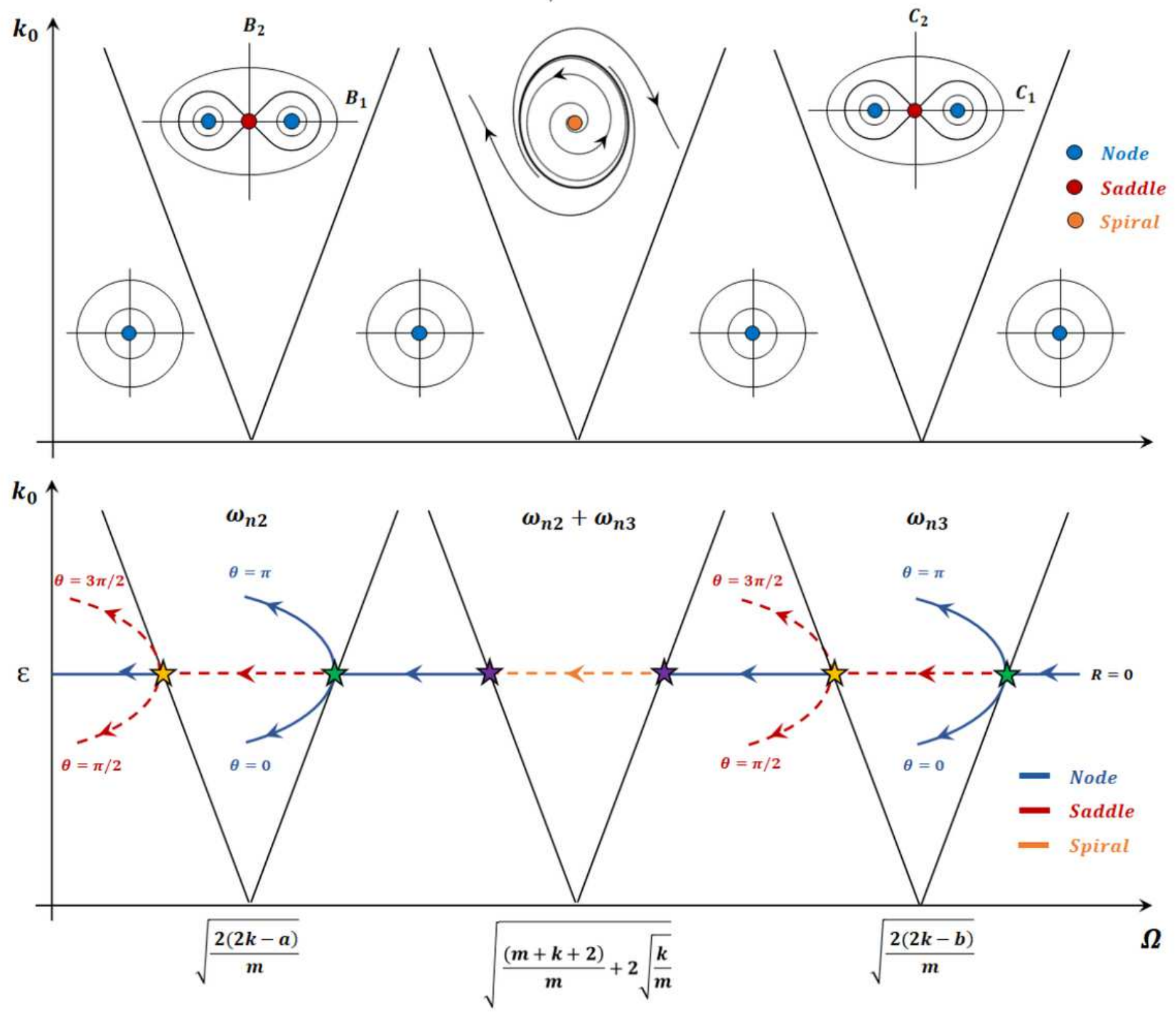

Figure 10

Global Bifurcation Diagram for the Softening Nonlinearity 

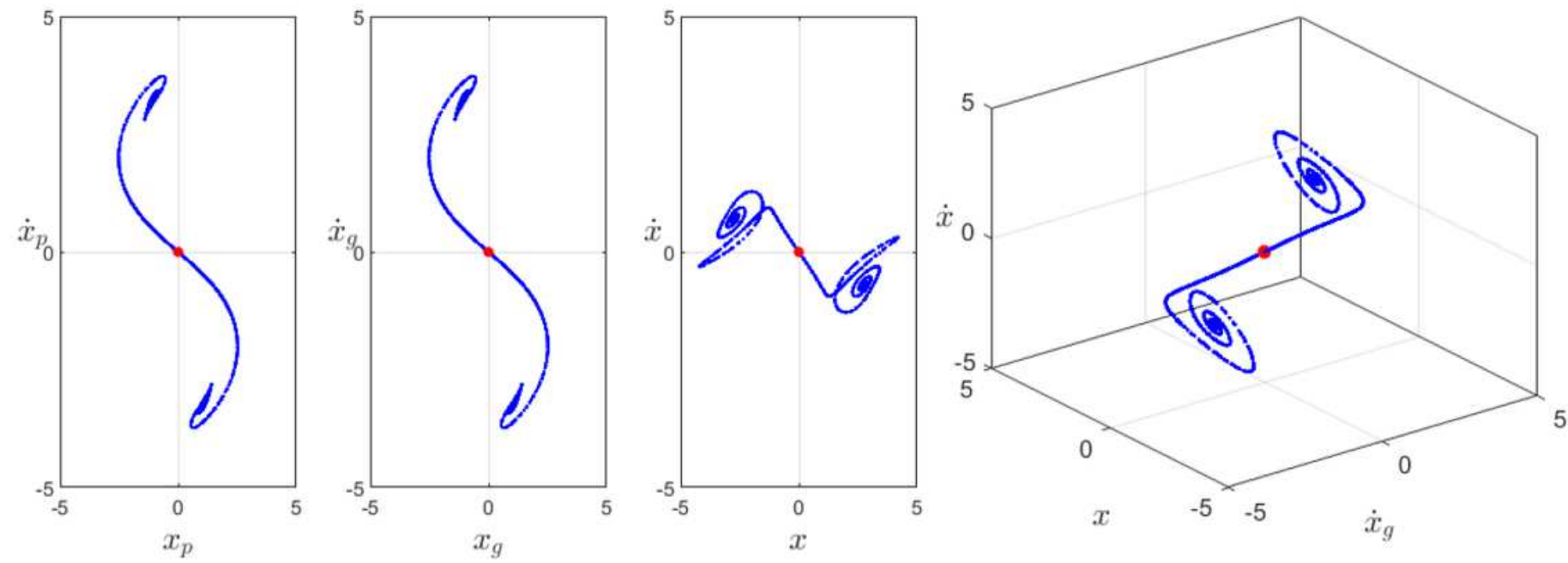

Figure 11

Poincare map softening, $m=1 ; \zeta=0: 0520 ; k=5 ; a=-0: 2 ; k 0=0: 5 ; \omega=1: 50$
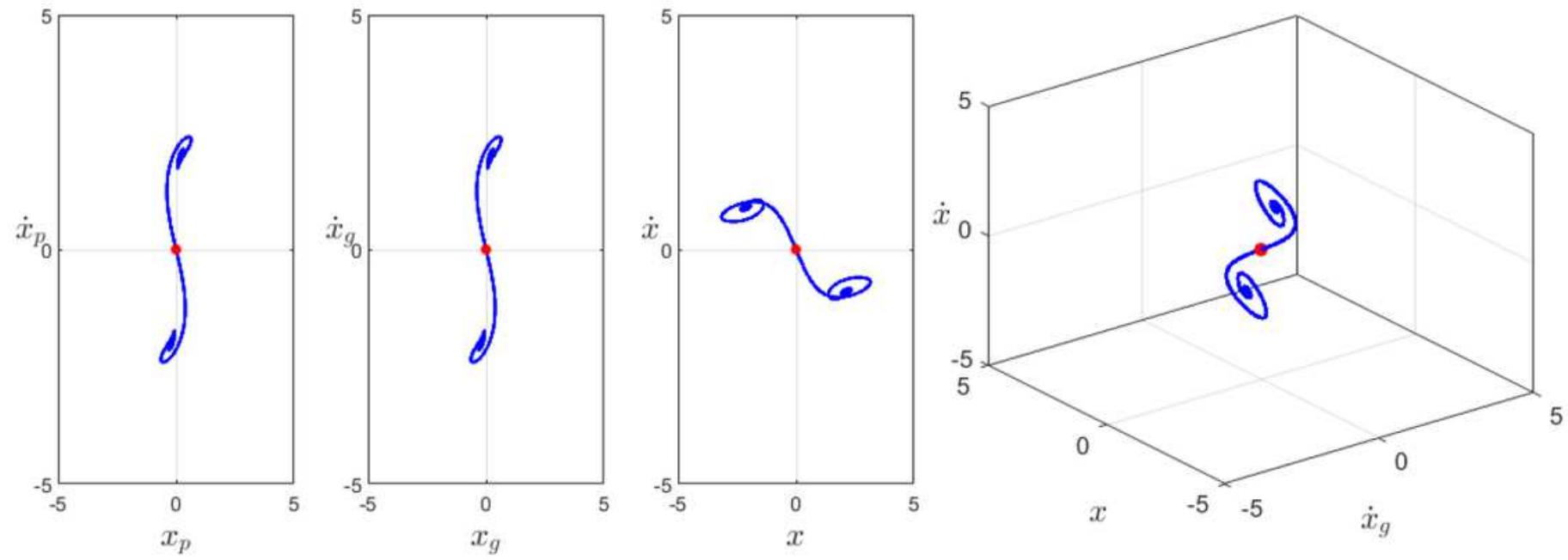

Figure 12

Poincare map, softening, $m=1 ; \zeta=0: 0300 ; k=5 ; a=-0: 2 ; k 0=0: 5 ; \omega=1: 64$ 

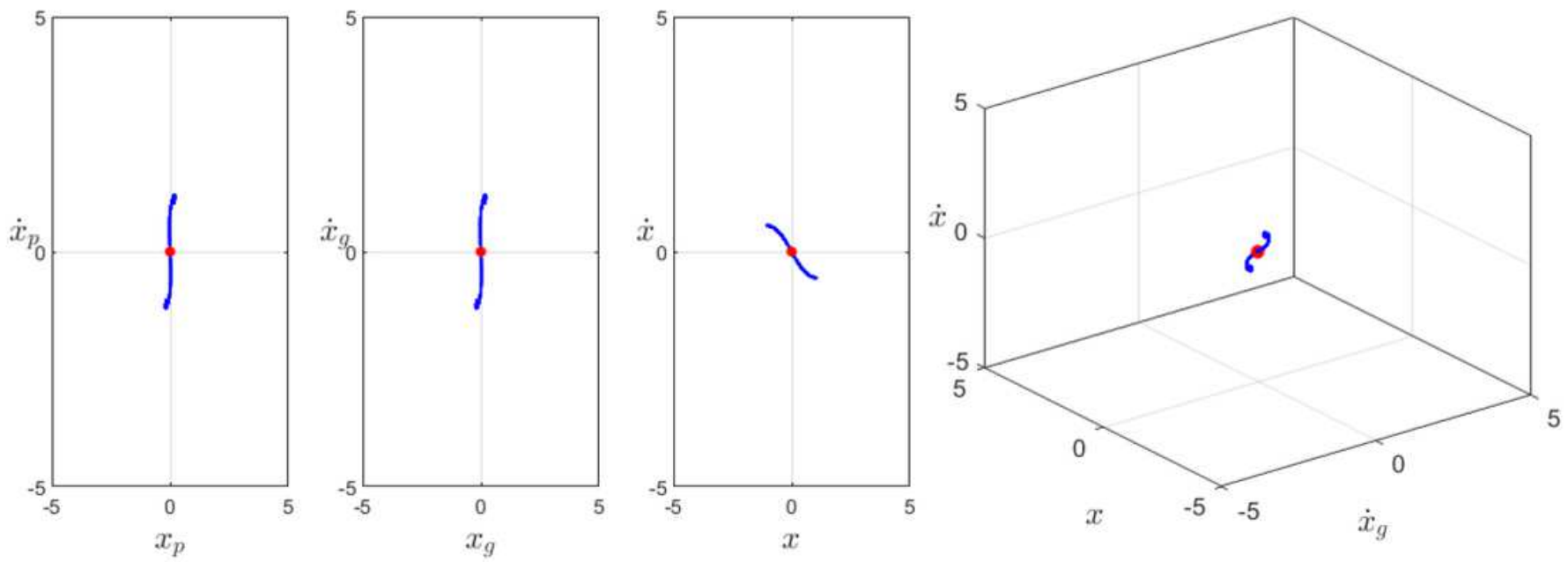

Figure 13

Poincare map, softening, $m=1 ; \zeta=0: 0200 ; k=5 ; a=-0: 2 ; k 0=0: 5 ; \omega=1: 68$
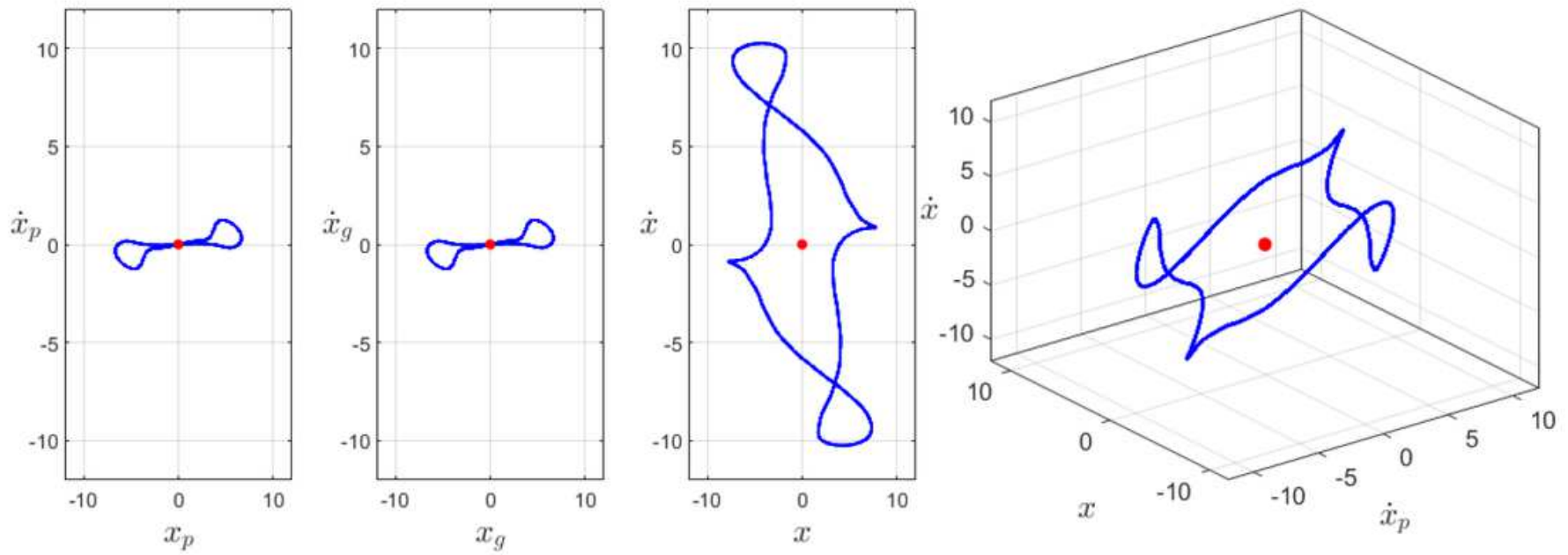

Figure 14

Poincare map, softening, $m=1 ; \zeta=0: 0100 ; k=5 ; a=-0: 2 ; k 0=0: 5 ; \omega=3: 50$ 

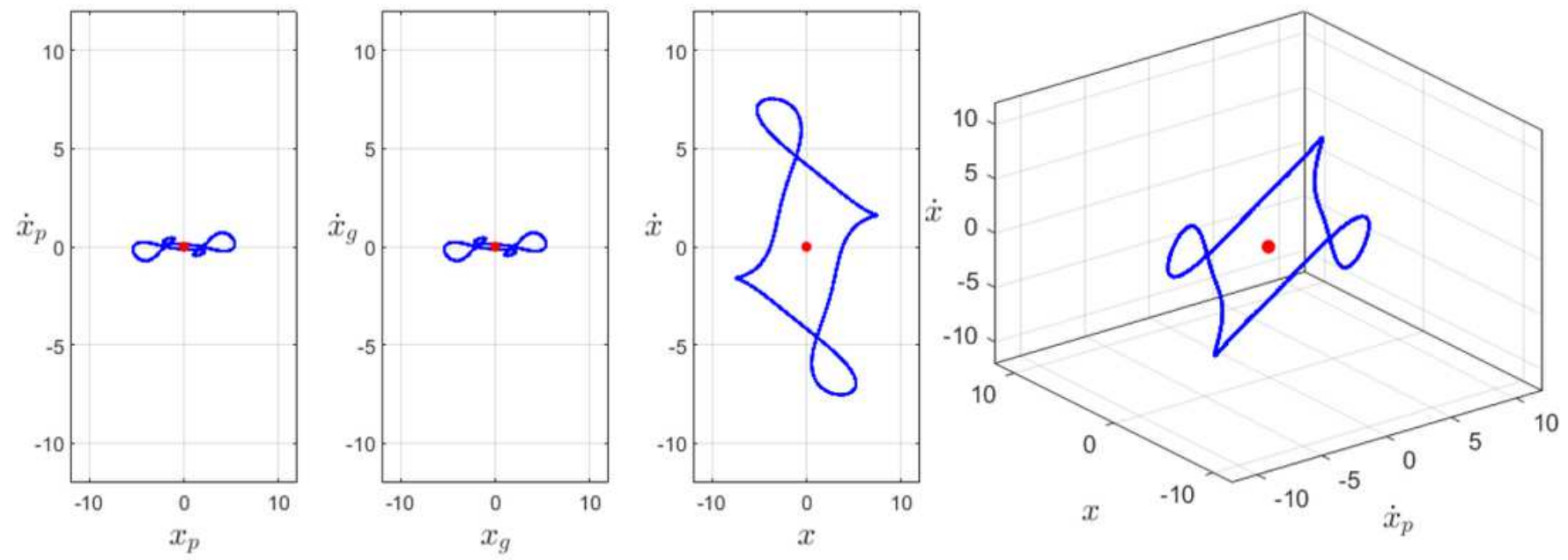

Figure 15

Poincare map, softening, $m=1 ; \zeta=0: 0100 ; k=5 ; a=-0: 2 ; k 0=0: 5 ; \omega=3: 60$
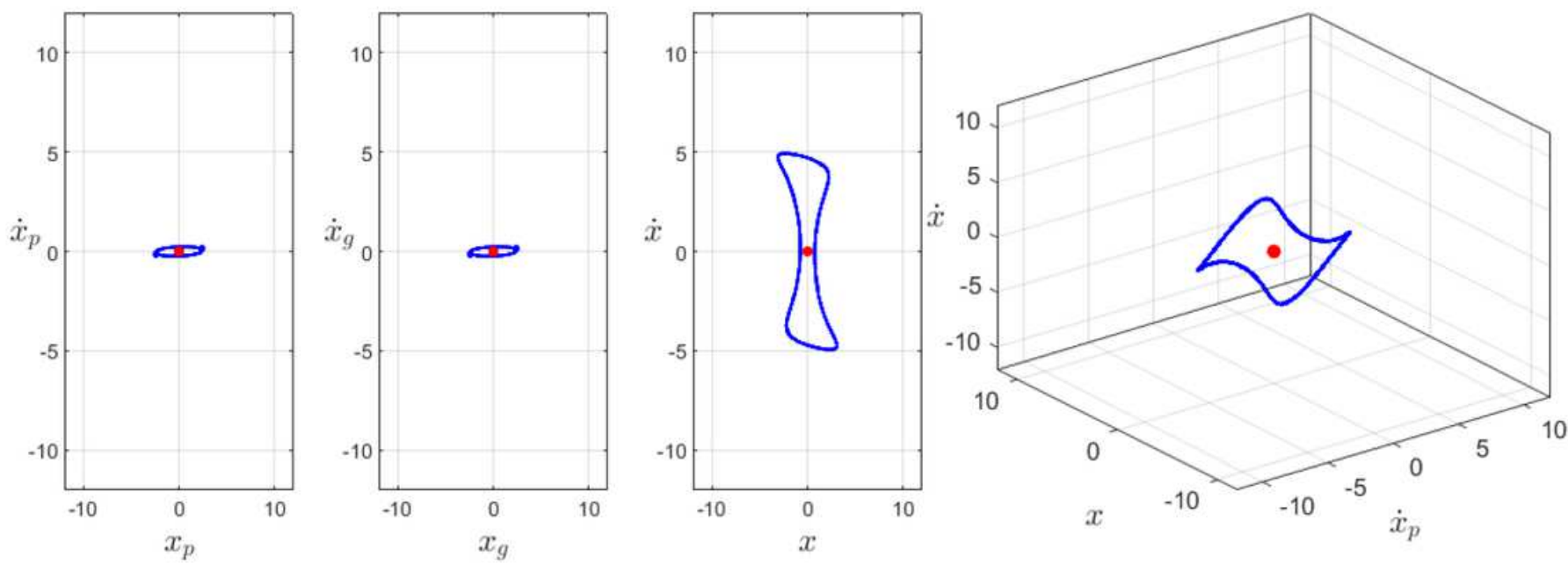

Figure 16

Poincare map, softening, $m=1 ; \zeta=0: 0200 ; k=5 ; a=-0: 2 ; k 0=0: 5 ; \omega=3: 70$ 

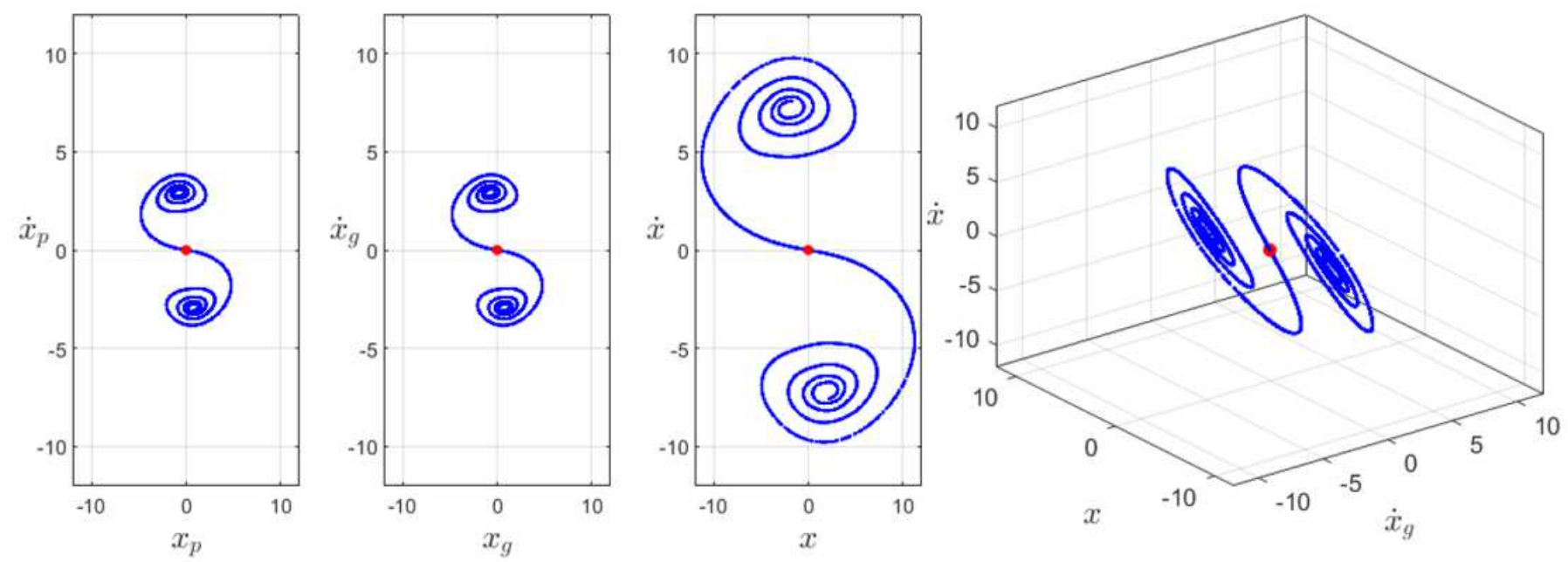

Figure 17

Poincare map, softening, $m=1 ; \zeta=0: 0075 ; k=5 ; a=-0: 2 ; k 0=0: 5 ; \omega=5: 20$
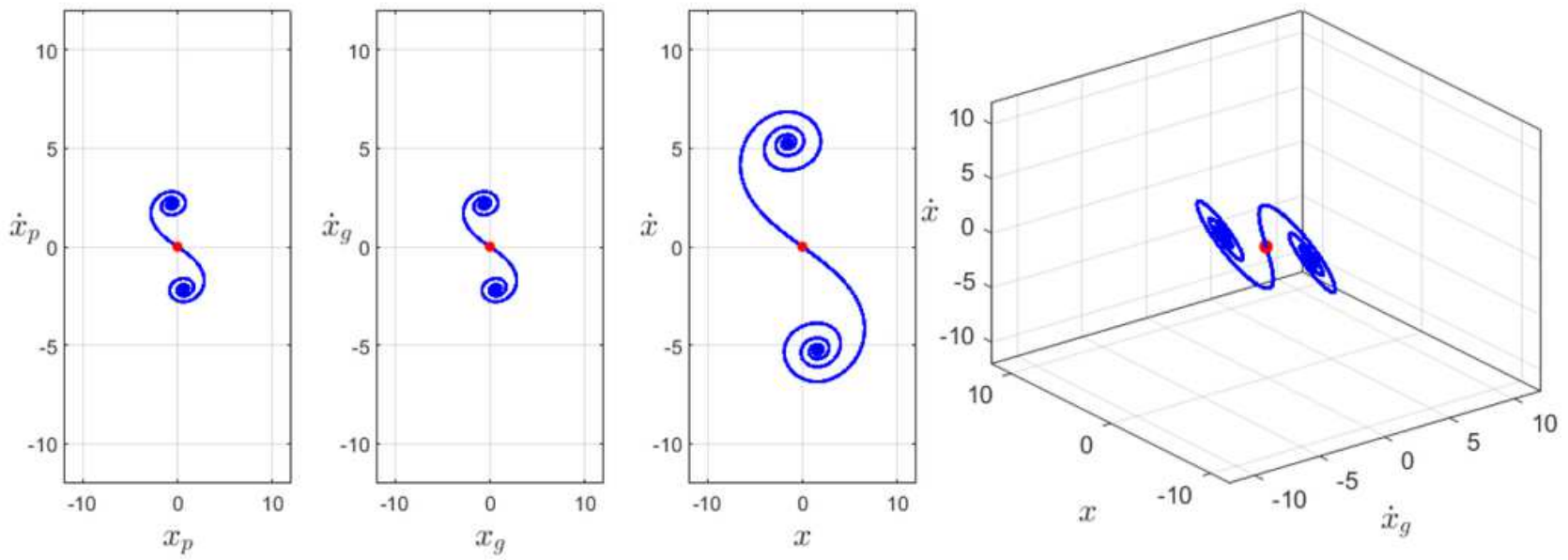

Figure 18

Poincare map, softening, $m=1 ; \zeta=0: 0075 ; k=5 ; a=-0: 2 ; k 0=0: 5 ; \omega=5: 40$ 

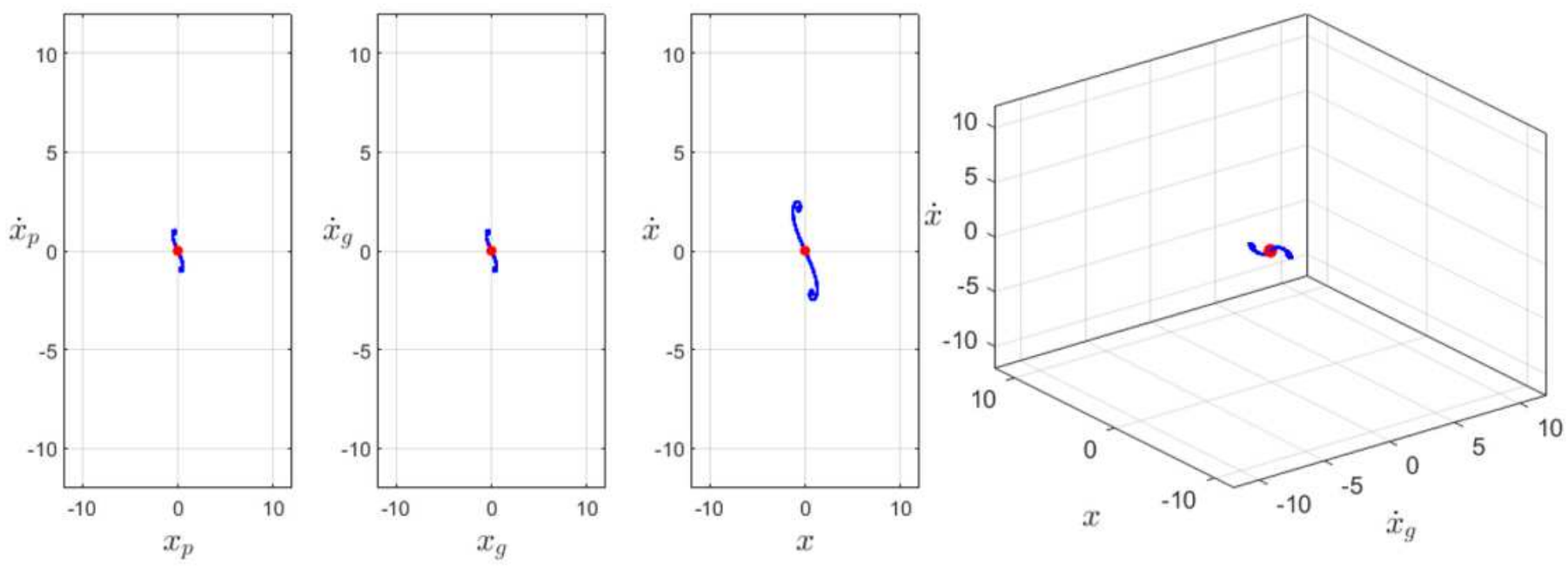

Figure 19

Poincare map, softening, $m=1 ; \zeta=0: 0075 ; k=5 ; a=-0: 2 ; k 0=0: 5 ; \omega=5: 60$ 

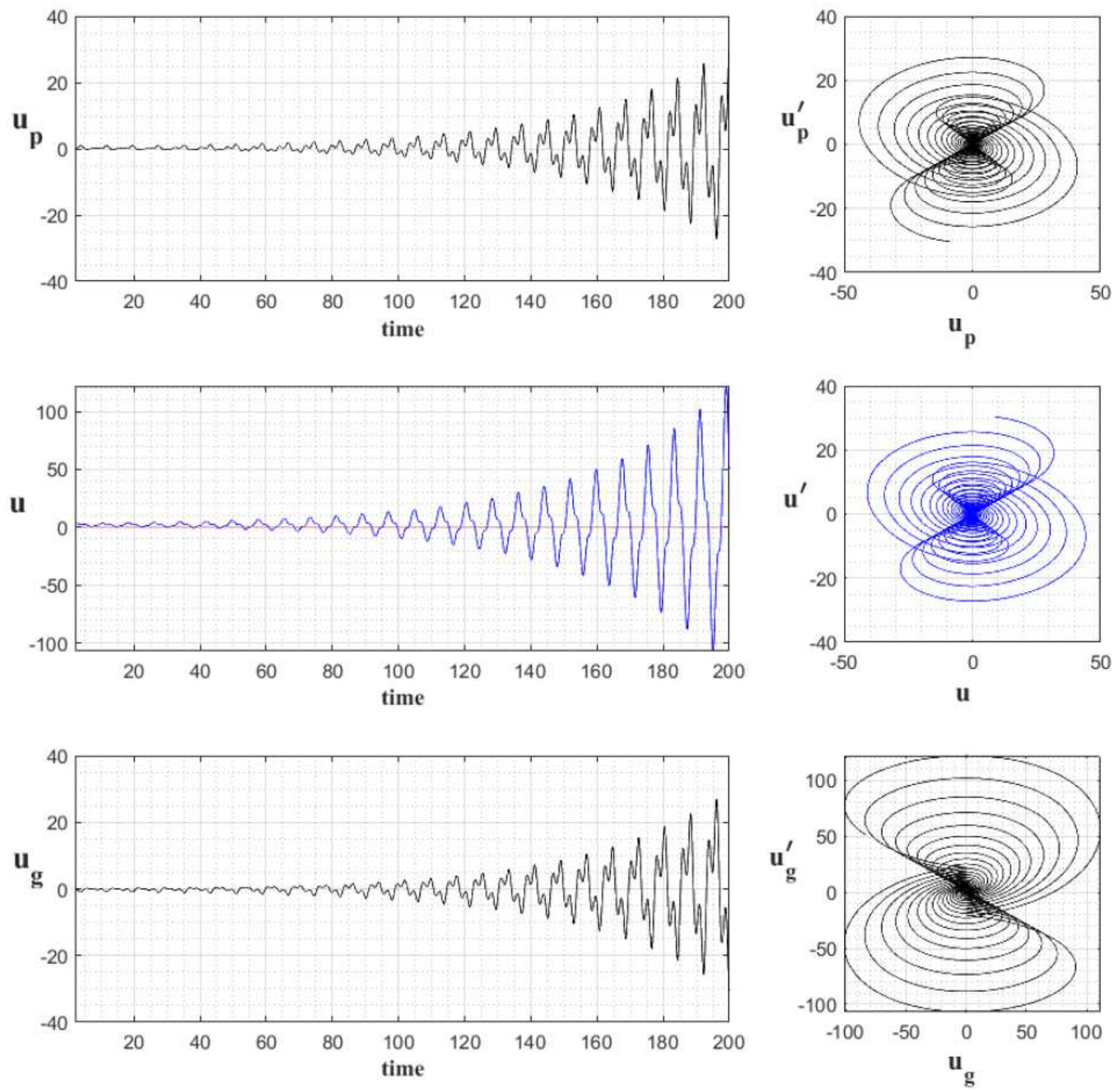

Figure 20

Time Response, $m=1 ; \zeta=0: 07 ; k=5 ; a=0 ; k 0=0: 5 ; F=1 ; \omega=1: 60$ 

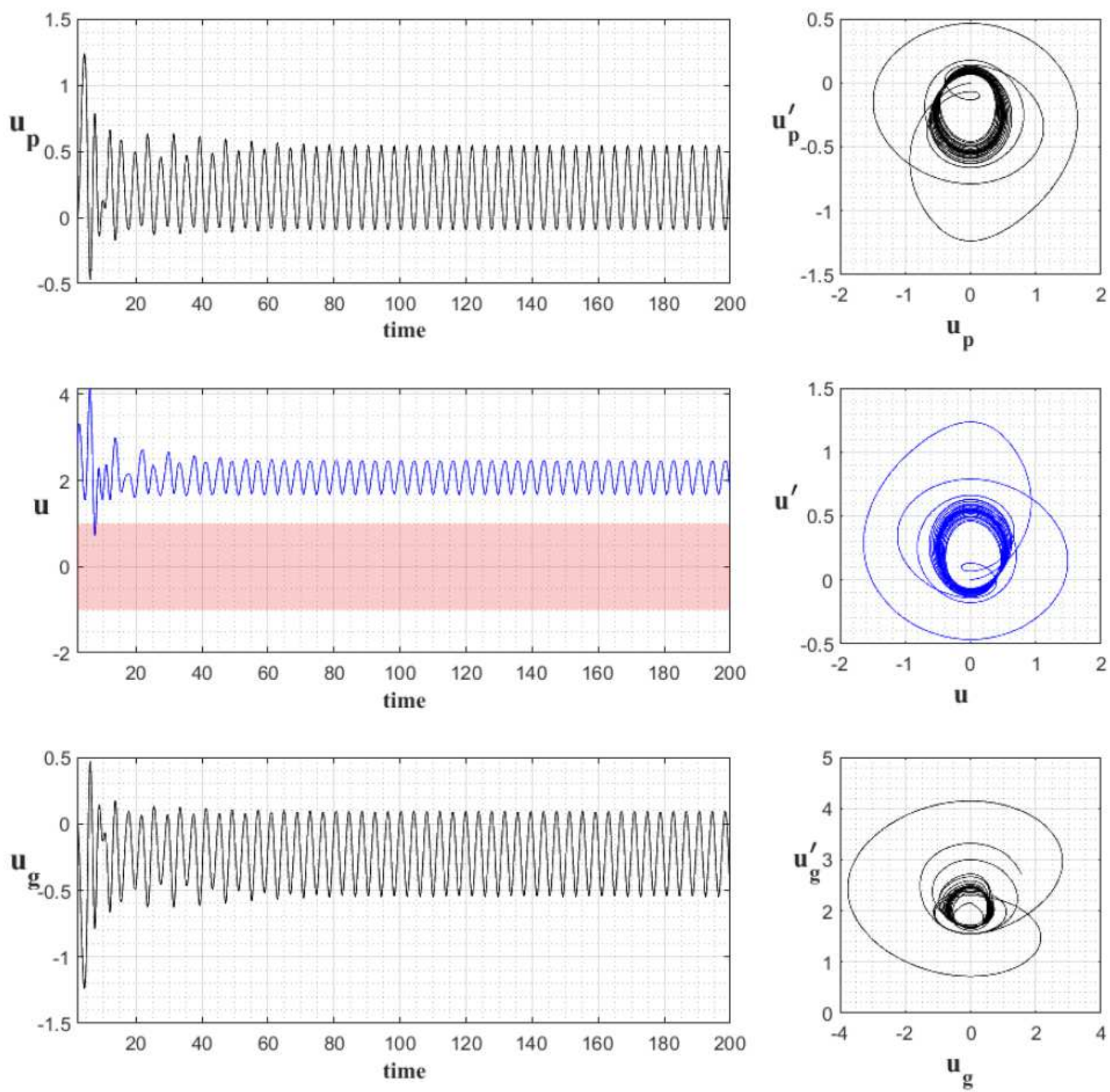

Figure 21

Time Response, $m=1 ; \zeta=0: 07 ; k=5 ; a=-2 ; k 0=0: 5 ; F=1 ; \omega=1: 60$ 